\title{
THE
}

\section{Predicting human cooperation in the Prisoner's Dilemma using case-based decision theory}

Todd Guilfoos

University of Rhode Island, guilfoos@uri.edu

Andreas D. Pape

Follow this and additional works at: https://digitalcommons.uri.edu/enre_facpubs

The University of Rhode Island Faculty have made this article openly available.

Please let us know how Open Access to this research benefits you.

This is a pre-publication author manuscript of the final, published article.

Terms of Use

This article is made available under the terms and conditions applicable towards Open Access Policy Articles, as set forth in our Terms of Use.

\section{Citation/Publisher Attribution}

Guilfoos, T., Pape, A.D. Predicting human cooperation in the Prisoner's Dilemma using case-based decision theory. Theory Decis 80, 1-32 (2016). https://doi.org/10.1007/s11238-015-9495-y

Available at: https://doi.org/10.1007/s11238-015-9495-y

This Article is brought to you for free and open access by the Environmental and Natural Resource Economics at DigitalCommons@URI. It has been accepted for inclusion in Environmental and Natural Resource Economics Faculty Publications by an authorized administrator of DigitalCommons@URI. For more information, please contact digitalcommons-group@uri.edu. 
Noname manuscript No.

(will be inserted by the editor)

\title{
Predicting Human Cooperation in the Prisoner's Dilemma Using Case-based Decision Theory
}

Todd Guilfoos • Andreas Duus Pape

Received: date / Accepted: date

\begin{abstract}
In this paper we show Case-based Decision Theory (Gilboa and Schmeidler, 1995) can explain the aggregate dynamics of cooperation in the repeated Prisoner's Dilemma, as observed in the experiments performed by Camera and Casari (2009). Moreover, we find CBDT provides a better fit to the dynamics of cooperation than does the existing Probit model, which is the first time such a result has been found. We also find that humans aspire to a payoff above the mutual defection outcome but below the mutual cooperation outcome, which suggests they hope, but are not confident, that cooperation can be achieved. Finally, our best-fitting parameters suggest that circumstances with more details are easier to recall. We make a prediction for future experiments: if the repeated PD were run for more periods, that we would be begin to see an increase in cooperation, most dramatically in the second treatment, where history is observed but identities are not. This is the first application of Case-based Decision Theory to a strategic context and the first empirical test of CBDT in such a context. It is also the first application of bootstrapped standard errors to an agent-based model.
\end{abstract}

JEL codes: D01, D83, C63, C72, C88

Keywords Case-based Decision Theory · Prisoner's Dilemma · Learning · Agent-based Computational Economics · Experimental Economics

The order of the authors is not indicative of effort or contribution towards this article.

T. Guilfoos

Department of Environmental and Natural Resource Economics, University of Rhode Island, 219 Coastal Institute, 1 Greenhouse Road, Kingston, RI 02881

E-mail: guilfoos@mail.uri.edu

A. D. Pape

Economics Department, Binghamton University, PO Box 6000, Binghamton NY, 13902

Phone: 607-777-2660 Fax: 607-777-2681

E-mail: apape@binghamton.edu 


\section{Introduction}

In this paper, we use Case-based Decision Theory (Gilboa and Schmeidler, 1995) to explain experimental data of human behavior in the repeated Prisoner's Dilemma game. We find that the aggregate dynamics of cooperation are predicted by this theory. We fit the parameters of the model to data and establish that all parameters are statistically significant. We establish this fact by comparing experimental data collected by Camera and Casari (2009) against simulated data generated by a computer program called the Case-based Software Agent (CBSA). CBSA was introduced in Pape and Kurtz (2013), who show that CBSA (and therefore Case-based Decision Theory) explains individual human behavior in a series of classification learning experiments from Psychology starting with Shepard, Hovland, and Jenkins (1961). Here we show that CBSA can explain human group behavior in a setting that is dynamic and strategic.

Case-based Decision Theory is a mathematical model of choice under uncertainty which has the following primitives: A set of problems or circumstances that the agent faces; a set of actions that the agent can choose in response to these problems; and a set of results which occur when an action is applied to a problem. Together, a problem, action, and result triplet is called a case, and can be thought of as one complete learning experience. The agent has a finite set of cases, called a memory, which it consults when making new decisions. The Case-based Software Agent is a software agent, i.e. "an encapsulated piece of software that includes data together with behavioral methods that act on these data (Tesfatsion, 2006)." CBSA computes choice data consistent with an instance of CBDT for an arbitrary choice problem or game, provided that the problem is well-defined and sufficiently bounded.

We analyze data from an experiment by Camera and Casari (2009), in which study subjects are grouped into small 'economies' to play the repeated Prisoner's Dilemma. The purpose of their experiment is to vary the level of information available to players about each other and measure the effect on cooperation. For example, in one treatment, the players are supplied unique identifiers for their opponents, so they know when they encounter the same opponent again. Because CBDT encodes the agent's information about the current choice directly (in the aforementioned "problem" variable), this is a particularly appropriate experiment to test with CBSA. We compare simulated data to real data by measuring the mean squared difference in probability of cooperation over time. Like regression analysis, we then search the space of free parameter values for those that provide the best fit (minimizing mean squared error). ${ }^{1}$ Moreover, we establish the precision by which we are able to estimate these parameters by bootstrapping standard errors, which is a first for agent-based models.

We are able to establish four key facts about CBDT and its relationship with human choice behavior in Camera and Casari's Prisoner's Dilemma experiment. We find:

(1) The choice behavior of this software agent (and therefore Case-based Decision Theory) correctly predicts the empirically observed trajectory of average cooperation rates over time across three different treatments. This shows that CBDT can predict human behavior in a strategic and dynamic setting.

(2) The choice behavior implied by CBSA is a closer fit to the empirical data than the best-fitting Probit model (from Camera and Casari's paper), and CBSA has only a fifth as many parameters to fit to data. This is a vote in favor of CBSA as a useful empirical description of human behavior in the repeated Prisoner's Dilemma and is a novel result in the literature.

(3) The best-fitting CBSA parameters suggest humans aspire to a payoff value above the mutual defection payoff but below the mutual cooperation payoff, which suggests they hope, but are not confident, that cooperation can be achieved. In principle, the best-fitting aspiration values could have fallen into

1 The free parameters of CBSA include two kinds of forgetfulness and an aspiration level. See Section 3 for details. 
the 'unreasonable' range: namely greater than the best or lower than the worst possible payoff. The fact that this did not happen serves as an specification test of CBSA. ${ }^{2}$

(4) Circumstances with more details are easier to recall. The evidence is that our best-fitting level of recall probability increases as the experimental treatment varies as to share more information with the agents.

These findings are useful in understanding the behavior of human subjects as well as developing a framework in which we can predict human behavior. For example, the infinitely iterated prisoner's dilemma can sustain cooperation when sufficiently-patient agents employ the 'grim' strategy, defecting forever if their partner defects, but this strategy does not seem to be played by human subjects. This paper can be thought of as part of an effort to find alternative, empirically valid explanations of decisionmaking in this strategic context.

Below, we first review the relevant literature in decision theory, game theory, and the empirical study of the Prisoner's Dilemma (Section 2). Second, we describe the experiment of Camera and Casari (2009) and explain how we simulate this experiment it in the Case-based Software Agent framework, which implements Case-based Decision Theory (Section 3). Third, we describe our statistical method of finding the best-fitting parameters of CBSA to match the human data, including how we bootstrap standard errors of our parameter estimates (Section 4). Fourth, we present and discuss our empirical results (Section 5), and, in Section 6, we discuss the implications of these results for case-based decision theory. In Section 7, we conclude.

\section{Related Literature}

The central investigative tool of this paper is the Case-based Software Agent (CBSA). It is a computational implementation of Case-Based Decision Theory (CBDT) introduced in Gilboa and Schmeidler (1995). Implementations produce agent choice behavior given a mathematical representation in the tradition of von Neumann and Morgenstern (1944) and Savage (1954). Designed correctly, the choice behavior produced by an implementation can be directly compared to empirical choice data of the same problem faced by humans. This can yield two classes of insights: First, the comparison can shed light on the question of whether and in what ways CBSA (and therefore CBDT) serves as a representation or 'explanation' of human behavior. Second, the comparison can shed light on the empirical phenomenon itself: for example, we learn what level of forgetfulness is consistent with the human behavior observed in the experiments found in Camera and Casari (2009).

CBDT postulates that when an agent is confronted with a new problem, she asks herself: How similar is today's case to cases in memory? What acts were taken in those cases? What were results? She then forecasts payoffs of actions using her memory, and chooses the action with the highest forecasted payoff. The primitives are: a finite set of problems $\mathcal{P}$, a finite set of acts $\mathcal{A}$, and a set of results $\mathcal{R}$. A case is a triplet consisting of a problem, the act taken, and the outcome (result) of that act given the problem. A case can be thought of as a single, complete learning experience. The set of all cases is $\mathcal{C}=\mathcal{P} \times \mathcal{A} \times \mathcal{R}$.

CBDT representations are defined by four additional components. The first component of a CBDT representation is the agent's memory. Memory is a set of cases which, in CBSA, can be thought of as the list of learning experiences the agent has had. An agent's memory is denoted $\mathcal{M}$. The second component is the utility function $u: \mathcal{R} \rightarrow \mathbb{R}$. It is defined in the usual way. The third component is the similarity

\footnotetext{
${ }^{2}$ Like other specification tests, passing the test does not mean that the model is necessarily correctly specified; only that failing the test would have been evidence that it is misspecified.
} 
function $s: \mathcal{P} \times \mathcal{P} \rightarrow[0,1]$. The output value of the similarity function gives how much the input problems resemble each other in the opinion of the agent. The fourth component is the aspiration level $H \in \mathbb{R}$. It is a reference level of utility, like expected value. However, while an expected value is the level of utility one believes on average is the most likely, the aspiration level should be thought of as the agent's target level of utility, which could, in principle, differ from the expected value. Mechanistically, it serves as a default value for forecasting utility of new alternatives. It also serves as a satificing level in the sense of Simon (1957): "Behaviorally, $H$ defines a level of utility beyond which the [decision maker] does not appear to experiment with new alternatives (Gilboa and Schmeidler, 1996, page 2)."

Together, these four components define case-based utility:

$$
C B U(a)=\sum_{(q, a, r) \in \mathcal{M}(a)} s(p, q)[u(r)-H]
$$

Where $\mathcal{M}(a)$ is defined as the subset of the agents' memory $\mathcal{M}$ in which action $a$ was taken. This utility represents the agent's preference in the sense that, for a fixed memory $\mathcal{M}, a$ is strictly preferred to $a^{\prime}$ if and only if $C B U(a)>C B U\left(a^{\prime}\right)$.

Case-based Decision Theory was introduced for the main purpose of disposing of the state space: that is, the assumption that agents are able to list and reason about the set of all possible scenarios. CBDT limits the set the agent must reason about to the set of past experiences, and requires only that the agent be able to make similarity judgements between past experiences and new experiences. Therefore CBDT naturally incorporates cognitive constraints. Moreover, the implementation of CBSA here in this paper also includes forgetfulness explicitly. ${ }^{3}$

This paper contributes to a growing empirical literature testing the explanatory power of CBDT; these papers generally find support for it.

In the paper most closely related to this one, Pape and Kurtz (2013) introduce CBSA and find that imperfect memory, accumulative (not average) utility, a similarity function consistent with research from psychology, and a $80-85 \%$ target success rate renders CBSA a good fit for human data in the classification learning experiment from the psychology literature. ${ }^{4}$

Ossadnik, Wilmsmann, and Niemann (2012) run a repeated choice experiment involving unknown proportions of colored and numbered balls in urns, which is the canonical ambiguous choice setting (i.e. Ellsberg, 1961). (The authors of CBDT suspect that it is a better model of human behavior in settings of ambiguity versus risk.) Ossadnik et al (2012) find that CBDT explains these data well compared to alternatives such as minimax (Luce and Raiffa, 1957) and reinforcement learning (Roth and Erev, 1995a). Their method has some similarities with CBSA, in that they choose parameter values and functional forms of CBDT and calculate CBDT-governed agents' optimal choices, and compare those choices to aggregate human data. There are two important ways that the method differs from CBSA. First of all, the CBSA method sweeps parameter values, so provides many more candidate values for fitting the human data. Second, CBSA integrates forgetfulness and similarity functional forms from psychology.

Bleichrodt, Filko, Kothiyal, and Wakker (2012) provide a method to measure similarity weights which avoids parametric assumptions about the weights. Their method has a number of advantages, including testing CBDT in more generality. An advantage of the CBSA approach is the ability to predict CBDTgoverned behavior on arbitrary settings; the Bleichrodt et al (2012) approach implies a particular kind

\footnotetext{
${ }^{3}$ Incorporating cognitive constraints into economic models is a hallmark of work in the intersection of economics and psychology, such as Simon (1957), Simon, Egidi, and Marris (2008), Tyson (2008), Hanoch (2002), Ballinger, Hudson, Karkoviata, and Wilcox (2011), and Cappelletti, Guth, and Ploner (2011).

${ }^{4}$ In particular, the 'SHJ' series of classification learning experiments, starting with Shepard, Hovland, and Jenkins (1961) and including Nosofsky (1986) and Nosofsky et al (1994).
} 
of experimental design. Therefore, we feel these methods are complementary: insights developed in their method can be applied to CBSA for application to other settings.

Gayer, Gilboa, and Lieberman (2007) investigate whether case-based reasoning appears to explain human decision-making using housing sales and rental data. They hypothesize and find that sales data are better explained by rules-based measures because sales are an investment for eventual resale and rules are easier to communicate, while rental data are better explained by case-based measures because rentals are a pure consumption good where communication of measures is irrelevant.

Golosnoy and Okhrin (2008) use CBDT to construct investment portfolios from real returns data and compare the success of these portfolios to investment portfolios constructed from expected-utility-based methods, and find some evidence that using CBDT aids portfolio success.

The Prisoner's Dilemma (PD) is perhaps the most famous game in game theory. It is a symmetric, simultaneous, two-player game with two actions, Cooperate and Defect, where (1) Defect strictly dominates Cooperate, but (2) the payoff for (Cooperate, Cooperate) Pareto dominates (Defect, Defect). Although there are benefits to cooperation, the individual incentive to defect means that mutual cooperation is not a Nash Equilibrium. Instead, (Defect, Defect) is the unique Nash Equilibrium. Because of this tension between what is best for the group versus the individual, the repeated Prisoner's Dilemma is used as a metaphor for cooperation in general and has been used to represent situations including public goods problems, common pool resource depletion, and negotiation in international politics.

Much of the theoretical investigation into the repeated Prisoner's Dilemma has been about the question: when does cooperation occur and when is it sustainable? There are many reasons why individuals might choose to cooperate, such as reputation building, altruism, or fear of reprisal. The fear of reprisal was formalized in the famous Folk Theorem first suggested by Friedman (1971), where players cooperate in the infinitely repeated Prisoner's Dilemma in a sustainable (i.e. subgame perfect Nash) equilibrium. The reprisal the players fear is that their opponent will defect in all future periods, therefore reverting to the suboptimal Nash outcome. This has been shown to be stable when players are sufficiently patient (Fudenberg and Maskin, 1986). However, this does not imply that rational agents will necessarily cooperate, as mutual defection in all periods is also a subgame perfect Nash equilibrium.

Experimentalists have also been investigating the causes and sustainability of cooperation through different treatments in experiments for some time now. ${ }^{5}$ Most relevant to our investigation today, Camera and Casari (2009) show that punishment and information of past play history can lead to higher sustainable levels of cooperation. They show this by experimentally varying the level of information available to players and measuring the resulting levels of cooperation. We attempt to explain their data with CBSA.

The central investigative tool of this paper is a software agent, therefore this paper is part of Agentbased Computational Economics. There is a long history of using computational agents to explore the repeated Prisoner's Dilemma (PD): most famously, Axelrod (1980) ran a series of tournaments where academics and computer programmers submitted strategies that play against each other in a repeated

5 The experimental literature on the PD and other repeated games is vast. A sample follows; also see the discussion in Section 6. Brosig (2002) show that signaling in face-to-face experiments may be effective at encouraging cooperation. In one-shot games there exists a low level of cooperation (Bereby-Meyer and Roth, 2006). Agents may learn to cooperate in repeated games, especially when monitoring of other players actions is allowed (Selten and Stoecker, 1986; Andreoni and Miller, 1993; Hauk and Nagel, 2001) but that cooperation breaks down during the course of the game. Evidence on the altruism motivation is mixed, some finding evidence for (e.g. Kreps et al, 1982) and some against (e.g. Cooper et al, 1996). Other papers of note include Ellison (1994); Bó (2005) and Bo and Fréchette (2011). Chong et al (2006) and Camerer and Hua Ho (1999) use an 'experience weighted attraction' model to study learning in a repeated trust game. This model postulates that players remember the history of previous play and form beliefs about what other players will do in the future and also are reinforced by how successful previous strategies have been. Monterosso (2002) uses false feedback to disrupt equilibria and measures the effects; this work seems also well-suited for analysis by CBSA and analyzing these data are a possible future extension. 
PD in one of the earliest and most famous agent-based investigations. Similarly, Miller (1996) explores the evolution of strategies when computational agents pick from a predetermined set. They investigate which strategies survive over repeated play of the PD as information is varied. This strain of literature has typically involved agents following simple strategies that are tailor-made for this application, such as tit-for-tat or the grim trigger strategy. The contribution of CBSA to this literature, other than its striking empirical fit, is that CBSA is a general choice engine that can be used in other games and decision problems.

\section{The Camera and Casari (2009) Experiment and CBSA}

In this section we describe how to use the Case-based Software Agent (CBSA) to apply case-based decision theory to the experiment performed by Camera and Casari (2009). This involves recreating the environment of the experiment within the confines of the software as accurately as possible, so that a comparison between the simulated 'data' produced by CBSA and the human data is a test of case-based decision theory and not artifacts of the implementation or software. Because this is a simulation, every function and parameter must be specified for a particular run, these choices are specified below. After establishing this construction, the statistical method behind the comparison between CBSA and human data is discussed in Section 4.

Fig. 1: The Prisoner's Dilemma used in Camera and Casari (2009)

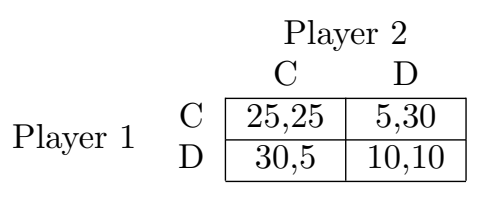

First, we describe the experiment performed by Camera and Casari. The specific parameterization of the Prisoner's Dilemma used by Camera and Casari is shown in Table $1 .{ }^{6}$ As usual, the first payoff listed is for player 1 and the second is for player 2. In the experiment, the human subjects are put into 4-person groups. A group is called an 'economy.' Each economy plays one 'supergame.' A 'supergame' is a series of PD games among the four players, played for a random number of periods. Each period, the 4 subjects are randomly paired to play PD. ${ }^{7}$ After both pairs play and payoffs are given, with a fixed probability $(1-\delta)$, the supergame immediately ends; and with continuation probability $\delta$ the game is played again. This repeats until continuation fails. Camera and Casari (2009) set $\delta=0.95$ which implies that at all times, the conditional expectation is that there will be 20 more periods of play.

Camera and Casari investigate four experimental treatments, and we consider three of these four treatments here. ${ }^{8}$ The treatments are designed to investigate how the level of anonymity influences players' decisions to cooperate. Increasing the level of information about other players' identities and past play expands the set of possible cooperative equilibria in the games. Correspondingly, Camera and Casari find that more public information of players' identities results in more cooperative behavior.

\footnotetext{
${ }^{6}$ See Camera and Casari (2009) for details about why these payoff values were selected.

7 Randomly paired with a uniform probability.

8 The excluded treatment involves an additional stage game after the PD is played. We hope to study this treatment in a future extension.
} 
Now we discuss the CBSA implementation of the Camera and Casari experiment. CBSA shares all primitives with CBDT, so there is a set of problems, a set of actions, and a set of results. A single learning experience, called a case, is a problem, action, and result triplet. In addition to these three primitives, CBSA follows case-based decision theory in defining a utility function, a similarity function, and an aspiration level, as well as an additional parameter: introduced here, CBSAs have an act randomization probability $\alpha \in[0,1]$. Finally, CBSA requires the definition of a so-called problem-result map or PRM. The PRM is the transition function of the environment. We define all these objects below.

The experience of the human subject is to repeatedly play the Prisoner's Dilemma, so we wish to consider one round of play as generating one learning experience or case. Correspondingly, the set of actions $\mathcal{A}$ is the set of actions in the one-shot Prisoner's Dilemma: $\mathcal{A}=\{C, D\}$, and the set of results $\mathcal{R}$ are the payoffs found in Table $1: \mathcal{R}=\{5,10,25,30\}$.

The set of problems is more complicated. The 'problem' can be thought of as the vector of information observable by the player before the choice is made. ${ }^{9}$ In the Camera and Casari setting, the experimental treatment is to vary the player's observable information: therefore, the problem set definition varies with the treatment. We define the problem set that corresponds to each of experimental treatments belowbut first we reason that in all treatments, players are aware of how far into the supergame they are, so all treatments' problem vectors include the period of play $t$. The other elements of the problem vectors vary by treatment.

Treatment 1 is private monitoring, which consists of anonymous subjects playing the supergame with no information about the player they are paired against or the players in the rest of the economy. Since no other information is available, $\mathcal{P}_{1}=T$, with typical element $p_{1}=(t)$, where $t \in T=\{1,2,3, \ldots\}$.

Treatment 2 is anonymous public monitoring, which gives information about the history of other players, including the highlighted history of one's current opponent. However, explicit identifiers are not available. In this treatment, we reason that the relevant information is the average cooperation rate of ones current opponent. So $\mathcal{P}_{2}=T \times[0,1]$, with typical element $p_{2}=\left(t, \bar{a}\left(\theta^{\prime}\right)\right)$. The dimension corresponding to the interval $[0,1]$ is the average cooperation rate of the opponent, where $\theta^{\prime}$ is the identity of the opponent, and where $\bar{a}\left(\theta^{\prime}\right)$ is the average cooperation rate of opponent $\theta^{\prime}$.

Treatment 3 is non-anonymous public monitoring, which consists of the information available in Treatment 2 as well as a unique ID of their opponent. We represent this as a vector of three binary variables $\left(i d_{1}, i d_{2}, i d_{3}\right)$, where at any time exactly one $i d$ variable is 1 and the others are 0 . Therefore $\mathcal{P}_{3}=T \times[0,1] \times\{0,1\} \times\{0,1\} \times\{0,1\}$, with typical element $p_{3}=\left(t, \bar{a}\left(\theta^{\prime}\right), i d\left(\theta^{\prime}\right)\right)$, where $\theta^{\prime}$ is the identity of the opponent, where $\bar{a}\left(\theta^{\prime}\right)$ is the average cooperation rate of opponent $\theta^{\prime}$, and where $i d\left(\theta^{\prime}\right)$ is a string of dummy variables that indicate the identity of opponent $\theta^{\prime}$.

A tangent about the choice of Camera and Casari for testing CBSA: The fact that the experimental treatment is to vary the information available to the players means that this setting is ideal for testing CBSA, and is, in fact, one of the key factors we considered in selecting this experiment to model and test. Since the treatments in the experiment imply different definitions of the set of problems $\mathcal{P}$, this experiment provides a set of a priori hypotheses that are empirically testable: that under the varying definitions of the set $\mathcal{P}$ defined by the treatments, CBSA's level of cooperation will move in tandem with humans. Defining the problem sets requires some interpretation; that is, the treatments do not uniquely identify corresponding problem sets. But, importantly, these problem set definitions were chosen without regard for goodness-of-fit (they were chosen before statistical analysis).

\footnotetext{
9 This is akin to the player's information set, but there is an important difference: the information set usually includes the history of play. That is not necessarily the case here, because 'history' is typically handled by the player's memory, that is, set of past learning experiences, and not the problem vector.
} 
We assume the utility function $u: \mathcal{R} \rightarrow \mathbb{R}$ is simply the risk-neutral $u(x)=x$; this means that we assume the payoffs of the game represent utility payoffs.

The similarity function $s: \mathcal{P} \times \mathcal{P} \rightarrow[0,1]$ describes how similar two circumstances are in the mind of the CBSA. Pape and Kurtz (2013) found that, consistent with the evidence from psychology (Shepard, 1987), that the similarity function has the following form, which was provided with an axiomatic foundation by Billot, Gilboa, and Schmeidler (2008). We assume this functional form here:

$$
\begin{aligned}
& s(p, q)=\frac{1}{e^{d(p, q)}} \\
& \text { where } p, q \in \mathcal{P} \\
& \text { and } d(p, q)=\sqrt{\sum_{i=1}^{\# \text { Dims }\left[\left(p_{i}-q_{i}\right)^{2}\right]}} \\
& \text { and } p_{i} \text { refers to the } i^{\text {th }} \text { element of } p
\end{aligned}
$$

The term "\# Dims" refers to the number of dimensions of the problem set $\mathcal{P}$ : \# $\operatorname{Dims}\left(\mathcal{P}_{1}\right)=1$, \# Dims $\left(\mathcal{P}_{2}\right)=2$, and \# Dims $\left(\mathcal{P}_{3}\right)=5$. It is plausible that different dimensions could have differing weights, that could plausibly vary over time. These are called 'attentional weights' in the psychology literature. We find these weights to be of little qualitative importance here so we do not consider them explicitly.

There is an aspiration level $H \in \mathbb{R}$, which represents a target level of utility of the agent. We explicitly consider this as a fitted parameter.

Along with the decision primitives, CBSA defines the decision environment: i.e. those parts of the choice problem that are external to the agent. ${ }^{10}$ In CBSA the decision environment is represented by a function (algorithm) called the problem-result map or $P R M$. The $P R M$ is the transition function of the environment. It takes as input the current problem $p \in \mathcal{P}$ the agent is facing, the action $a \in \mathcal{A}$ that the agent has chosen, and some vector $\theta \in \Theta$ of environmental characteristics. The $P R M$ returns the outcome of these three inputs: namely, it returns a result $r \in \mathcal{R}$; the next problem $p^{\prime} \in \mathcal{P}$ that the agent faces; and a potentially modified vector of environmental characteristics $\theta^{\prime} \in \Theta$. I.e.:

$$
P R M: \mathcal{P} \times \mathcal{A} \times \Theta \rightarrow \mathcal{R} \times \mathcal{P} \times \Theta
$$

In general in CBSA, $\theta$ describes the current state of the environment of each agent, i.e. exogenous, unknown forces that are acting on the agent. ${ }^{11}$ In the setting of Camera and Casari, the environment of the player is the identity of the opponent. Given this definition, the PRM finds the action $a(\theta)$ chosen by the opponent, then (1) assigns the payoff associated with actions $(a, a(\theta))$ to the result $r,(2)$ chooses a new opponent $\theta^{\prime}$, and (3) delivers the new problem vector $p^{\prime}$ associated with opponent $\theta^{\prime}$. For completeness, the explicit PRM is provided in Figure 7 (see Appendix B).

Figure 2 describes the choice algorithm which implements the core of CBSA, where the choice $C$ represents Cooperation and $D$ represents Defect. It is an algorithmic description of the choice process defined by CBDT, with two modifications. The modifications allow for imperfect memory. In Pape and Kurtz (2013), it was found that a match between CBSA and human data was only achieved by allowing for imperfect memory: otherwise CBSA solves the classification learning problem much faster than humans. We find that imperfect memory is also important for matching human data in this setting.

\footnotetext{
10 These need not be defined for a decision theory, so are not a formal part of CBDT, and need only be formally defined when one seeks to generate simulated choice behavior to compare to empirical data.

11 Known exogenous forces acting on the agent are part of the problem vector.
} 


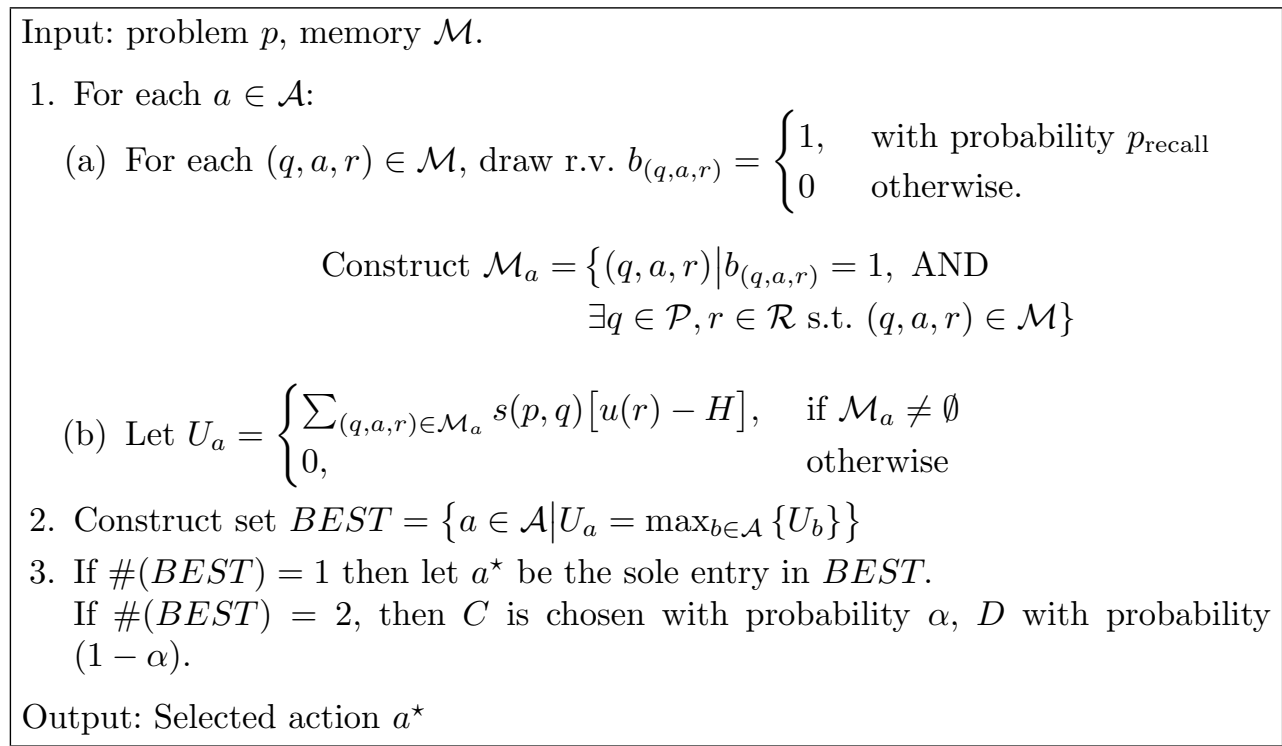

There are two kinds of imperfect memory. First, there is imperfect recall, governed by a probability $p_{\text {recall }} \in[0,1]$. Imperfect recall corresponds to an inability to access all memory at any given time, and it is therefore associated with limited cognitive capacity. Second, there is imperfect storage, governed by a probability $p_{\text {store }} \in[0,1]$. Imperfect storage corresponds to a failure to add some experiences to memory after they are experienced, and it is therefore associated with limited memory storage capacity.

In Figure 2, the agent faces a problem $p \in \mathcal{P}$ and has a memory $\mathcal{M} \subseteq \mathcal{C}$. In Step 1a, for each action $a$, she collects those cases in which she performed this act. Since her recall is imperfect, relevant cases are selected into the set $\mathcal{M}_{a}$ with probability $p_{\text {recall, }}$, where relevant cases which are not recalled are simply ignored. ${ }^{12}$ In Step 1b, she uses this subset of her memory $\mathcal{M}_{a}$ to construct a utility forecast of that act, called here $U_{a}$. The agent then chooses the action which corresponds to the maximum $U$. As seen in step 3 , when there is a tie between $C$ and $D, C$ is chosen with an exogenous probability $\alpha$. In the original formulation, it was assumed that $\alpha=.5$, but in this study we calibrate $\alpha$ to data (see Section 4 for calibration details).

Fig. 3: A Single Choice Problem.

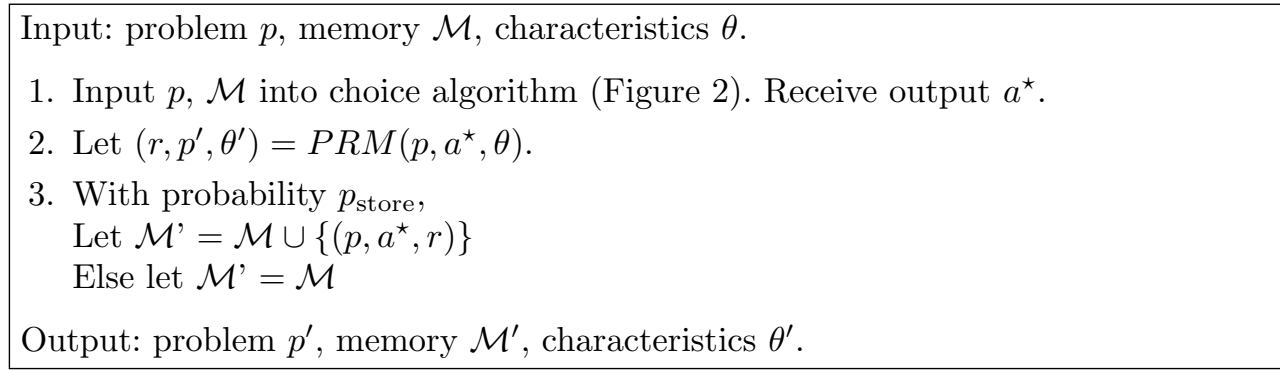

Figure 3 describes a single choice problem faced by the agent. It embeds a reference to the choice algorithm described in Figure 2. Figure 3 embeds the agent in an environment and explicitly references

\footnotetext{
12 When $p_{\text {recall }}=1$, the agent has perfect recall. It then corresponds to CBDT as it appears in Gilboa and Schmeidler (1995).
} 
that environment in the call to $P R M$. (The $P R M$ corresponding to Camera and Casari's experiment is described above and also appears in Appendix B.) In Step One of the single choice prolem the agent selects an act, $a^{\star}$. In Step Two, the action is performed in the sense that the environment of the agent reacts to the agent's choice: the $P R M$ takes the current problem $p$, the action $a^{\star}$ selected by the agent, and the characteristics unobserved by the agent $\theta$, and constructs a result $r$, a next problem $p^{\prime}$, and a next set of characteristics $\theta^{\prime}$. In Step Three, the agent's memory is augmented by the new case which was just encountered, so long as the agent does not have a 'write-to-memory error:' i.e., with probability $p_{\text {store }}$, the case that was just experienced is added to the set $\mathcal{M}$. With probability $\left(1-p_{\text {store }}\right)$, that case is discarded.

Since the choice problem depicted in Figure 3 maps a problem, a characteristic, and a memory vector to another vector in the same space, it can be applied iteratively. A series of such iterations, along with initial conditions and ending conditions, can then be used to produce a single time series of agent behavior, called a 'run.' The ending condition used by Camera and Casari, as mentioned above, is a $\delta$ probability of ending the supergame after each period. We follow this ending condition in the sense that we simulate the actual lengths of play that appear in the data; see Section 4 below for details.

\section{Statistical Model and Fitting Human Data}

In this section, we describe the statistical and simulation method by which we estimate the parameters of this model. First, we give an overview of the criteria we use to evaluate the explanatory power of the parameterized model and the process we use to estimate those parameters, which includes a description of the simulated data generation, the method we use to bootstrap standard errors for our parameters, and a description of these free and constrained parameters. Then we discuss the relevant psychometric literature, which is the source of this modeling perspective.

There are three criteria we use to evaluate the explanatory power of a model: qualitative fit, quantitative fit, and model complexity.

Qualitative fit is equivalent to matching "stylized facts" of human data. For example, we find that, empirically, Treatment 3 maintains a higher cooperation rate than Treatments 1 and 2 in all periods. A model which matches more of these regularities is said to have a greater qualitative fit.

Quantitative fit is a numeric evaluation of fit to human data: for any given set of simulated data, we construct Mean Squared Error (MSE) between the simulation average cooperation rate over time and human average cooperation rate over time. The lower the MSE, the better the quantitative fit. Even though perfect quantitative fit implies perfect qualitative fit, in practice quantitative fit can come at the cost of qualitative fit. In the selection of best-fitting parameter values, we search the parameter space to maximize quantitative fit and then evaluate qualitative fit of the best-quantitative-fit model.

Model complexity is known as 'over-fitting' in econometrics or 'model elegance' in theory. This third criterion rests on the observation that if a model is allowed to be arbitrarily complicated, then perfect qualitative and quantitative fit can be achieved, but such a model may be undesirable because it doesn't reveal insight into the phenomenon and is not generalizable out-of-sample. This consideration leads us to select a simpler model over a more complicated one. ${ }^{13}$ Like qualitative fit, model complexity is an ex-post evaluation criterion.

\footnotetext{
13 For another point of view on this criterion, consider two competing theories that attempt to explain the same phenomenon: theory $a$ and theory $b$. Suppose that there is some empirical data available about the phenomenon. Suppose theory $a$ has many more free parameters than theory $b$ does. Now suppose we calibrate theories $a$ and $b$ to the data, and we find, after calibration, that theory $a$ and theory $b$ both explain the same fraction of the variation in the data and explain the same qualitative phenomena. Then, under the model complexity criterion, theory $b$ is preferred.
} 
Quantitative fit guides the selection of best-fitting parameters in a manner analogous to linear regression. Like linear regression, we seek a set of parameters of a mathematical model that best fit observed data by minimizing mean squared error between predicted and observed values of the outcome variable. However, unlike regression, there is no known closed-form function from the observed data to the parameters of CBDT. ${ }^{14}$ Because there is no closed-form function, we search the space of parameter values by (1) running CBSA with these different parameter values, (2) generating simulated data, and (3) measuring those simulated data against the human data according to mean squared error (MSE), and returning to step 1 with new parameter values. Specifically, we explore the parameter space through an iterated grid-search: the parameter space is swept at a certain resolution, generating 1000 simulation runs for each parameter combination. Then, the part of the parameter space which contains the best fitting models is explored at a higher resolution, again with 1000 simulation runs per parameter combination. This is repeated until it appears we exhaust measurable improvements in MSE.

The 1000 simulation runs are generated in the following way: ${ }^{15}$ For each candidate set of parameter values, we construct 50 'sub-simulations,' each corresponding to one of the 50 observed supergames in the data. This is done by setting the number of periods and the random pairing in each period to that which occurred in the corresponding observed supergame. We then run each sub-simulation 20 times, generating $1000=20 \times 50$ total runs. Note that this means that we have 1, 000 period one observations, but as the rounds go on and some groups stop playing, the number of observations falls. By round 30 we typically have $2-3$ groups left in each treatment, both in the observed and simulated data. This corresponds to $200-300$ observations in the simulated data.

Beyond point estimates, we also desire to ascertain the statistical significance of our parameter estimates. We do this by bootstrapping standard errors using the data (Wooldridge, 2012). ${ }^{16}$ This process is similar to bootstrapping standard errors in a regression model: we randomly select a subset of the experimental panel data, select the best-fitting parameter values for that subset, and repeat, until we have generated a distribution for these parameter values. Given this constructed distribution of parameter values, we can construct standard errors of those estimates. Of course, here the best-fitting model is chosen for each subsample in the manner described above (1000 runs and comparing MSE).

The formula for the bootstrapped standard error of a parameter $\beta$ is

$$
S E(\beta)=\sqrt{\frac{1}{N-1} \cdot \sum_{i=1}^{N}\left(\beta_{i}-\hat{\beta}\right)^{2}}
$$

where $N$ is the number of times subsamples are drawn and parameters re-estimated, $\beta_{i}$ is the best fitting parameter estimate associated with the $i^{\text {th }}$ subsample, and $\hat{\beta}$ is the estimate of $\beta$ from the full data set.

There are three parameters of CBSA that are estimated in this manner. The first and second estimated parameters are the imperfect memory parameters: $p_{\text {store }}$, which is the probability that an individual case is written to memory, and $p_{\text {recall }}$, which is the probability that an individual case is recalled when memory is accessed. The third estimated parameter is the aspiration level $H$, which is the target payoff level sought by individuals. ${ }^{17}$

\footnotetext{
14 I.e. the equivalent to $\beta=\frac{X^{\prime} X}{X^{\prime} Y}$.

15 Thanks to an anonymous referee for this suggestion.

16 To the authors' knowledge, this is the first example of bootstrapping the standard errors of parameters in an agent-based model.

17 There are also parameters we do not choose to vary. For example, we do not vary the functional form of similarity: we choose only the 'accumulative' form of similarity over 'average similarity,' as average similarity is found in Pape and Kurtz (2013) to cause the counterfactual behavior of individuals believing actions to be irrelevant. We also do not vary from the functional form of inverse exponential weighted Euclidean distance. There is a strong empirical case for this functional
} 
In addition to the three estimated parameters, there are two that are chosen according to theory. The first parameter chosen in this way is the problem vector $p$, which was described above. The second is $\alpha$, the probability of cooperating when indifferent. A brief theoretical analysis proves that $\alpha$ will be equal to the population average cooperation level in the first round of play. The logic is: at the beginning of the supergame, all agents are indifferent between $C$ and $D$ and therefore randomize with probability $\alpha$. Therefore, a priori the first round of play will, on average, yield a fraction $\alpha$ of cooperators, which of course turns out to be true in the simulated data. ${ }^{18}$

This method (other than bootstrapping) was used in the economic literature in Pape and Kurtz (2013). In that paper, the authors use CBSA to evaluate CBDT's ability to explain human learning behavior in a set of concept learning experiments from psychology, using psychological statistical methods ('psychometrics'). In this literature, mechanistic or algorithmic models of human behavior are used to simulate data, which are compared to human behavioral data collected in the laboratory. They and we follow the method used in the work of Nosofsky, Gluck, Palmeri, McKinley, and Glauthier (1994), which advanced the statistics of this field; in their own words, "although previous researchers have discussed the ability of different models to account for qualitative aspects of [concept learning experimental] data, in this research we begin the process of quantitatively testing such models (Nosofsky et al, 1994, p. 354)." Like us, Nosofsky et al search the space of free parameters to minimize the sum of square deviations between the (average) predicted and (average) observed outcome variable. ${ }^{19}$ This method is widely accepted in psychology, which uses these kinds of models fitted to 'micro-level' experimental panel data using algorithmic models like ours. ${ }^{20}$ This method also bears some similarity to calibration of macroeconomic models (Kydland and Prescott, 1996): perhaps the most important difference between this method and macroeconomic model calibration is that macroeconomic models calibrate to time-series instead of panel data. We intend to investigate this relationship more thoroughly in future work.

A final comment on this method: matching the simulated average data to the average of the outcome variable of the data ignores variance of those data. It stands to reason that it may be valuable to count more heavily observed outcomes that have low variance in data. Here we are also able to follow Nosofsky et al (1994), who present a weighted mean squared error, where the weights are chosen to address this concern: "The weighted [mean squared error] is found by [averaging] the squared deviation between the predicted and observed error proportions weighted by $\frac{1}{\sigma^{2}}$, the inverse of the variance of each cell proportion[.] (Nosofsky et al, 1994, p. 362)" 21 We try estimation with weighted mean squared error, and, like Nosofsky et al, find it has little effect on the results. Therefore, we do not present these results here.

\section{Experimental Results}

In this section we present the results of two versions of CBSA and compare them to the results of the Probit found in Camera and Casari (2009) and a constrained alternative Probit formulation. The purpose of this comparison is to validate CBSA: we seek to empirically 'benchmark' CBSA against some

form in psychology, which is bolstered by the results found in Pape and Kurtz (2013). Please see that paper and Section 3 of this paper for details.

18 An alternative modeling choice is to posit a two types of agents: ones who always cooperate when indifferent and ones who always defect. Then one calibrates the relative size of the two populations to the known cooperation rate in the first round. This alternative modeling strategy does not make a large difference in the results so it is not presented here.

19 In the case of concept learning the outcome variable in question is the rate of misclassification of particular objects over time in a supervised learning environment.

20 E.g. Nosofsky and Palmeri (1996), Love, Medin, and Gureckis (2004), Kurtz (2007), and Vigo (2013).

21 They cite Bishop et al (1975) as a statistical source for this method. 
alternative explanatory model. We compare the four models by the model selection criteria described in the previous section: quantitative fit, qualitative fit, and model complexity. An overview of the results can be seen in Table 1. In this table, we provide parameter estimates for the two CBSA versions and degrees of freedom and goodness-of-fit (MSE) of all four available models. The predicted outcome variable, average cooperation rates over time, for all four models as well as the raw data are shown in Figure 4. We also organize these predicted outcome variables by treatment and include $95 \%$ confidence intervals (the gray area around the CBSA prediction) in Figure 5.

We are able to show (1) that the CBSA correctly predicts the empirically observed trajectory of average cooperation rates over time across different treatments, and (2) that the choice behavior implied by CBSA is a closer fit to the empirical data than either Probit model. We also find that the best-fitting parameters suggest (3) humans aspire to payoff value above the mutual defection outcome but below the mutual cooperation outcome, which suggests they hope but are not confident that cooperation can be achieved, and (4) circumstances with more details are easier to recall. We also predict that, if the experiments of Camera and Casari were run for more periods, that we would begin to see an increase in cooperation among the players.

Probit and CBSA methods with similar goals: the coefficient estimates of a Probit emerge from an analytical maximization of a likelihood function given the data, and the CBSA parameter estimates emerge from a computational maximization of a quantitative measure of fit to data. In this sense, they are both predictive methods calibrated to data. Therefore, one could think that, if CBSA compares favorably to the Probit along some goodness-of-fit metric, then perhaps CBSA should be considered more seriously as an empirical method. On the other hand, if CBSA compares unfavorably to the Probit, it should be considered less seriously.

Note that this does not imply we seek to accept Probit over CBSA or the reverse. This is because CBSA and Probit could both be true. Suppose both the Probit and CBSA were good fits to explain the empirical data. One explanation could be that CBSA explains human learning behavior, and the memory, similarity, and utility of CBSA together encode strategies that the Probit measures, even though CBSA does not explicitly represent strategies. ${ }^{22}$ Another explanation notes that Decision Theory is built on representation theorems: if behavior matches certain axioms, then a utility function, beliefs, etc., that represent that choice can be constructed. However, in decision theory, no representation theorem claims exclusivity: on the contrary, so long as the axiom sets of two representation theorems are not mutually exclusive, then the choice behavior can be represented by the structures in each theorem. So if the Probit and CBSA both seek to explain behavior in the 'representation theorem' sense, they could both be valid. ${ }^{23,24}$

This section proceeds in four parts: First, we describe the four models depicted here. Second, we compare the two CBSA models and the two Probit models along the dimensions of quantitative fit and model complexity using Table 1. Third, we compare the four models along the dimension of qualitative fit by using Figure 4. Steps two and three establish results (1) and (2) above, that CBSA fits well and

\footnotetext{
22 This is possible if one notes that these computational structures of CBSA could 'encode strategies' in the way that a computer program encodes program behavior.

23 Moreover, Matsui (2000) shows that Case-based Decision Theory and Expected Utility Theory can both represent the same choice behavior almost always. If the Probit can be likened to an expected utility perspective, then Matsui's result would suggest that both the Probit and CBSA could match.

24 It is interesting to consider running the models on each others' outcomes: what strategies does a Probit suggest that the CBSA has encoded? And, in the other direction, what CBSA parameters emerge when CBSA seeks to predict the implied Probit strategies? We intend to consider these questions in a future extension.
} 
Table 1: CBSA and Probit Regression Predicting Average Cooperation Rates

\begin{tabular}{cccc}
\hline \hline Benchmark & Constrained & Full & Constrained \\
CBSA & CBSA & Probit & Probit
\end{tabular}

Treatment 1

\begin{tabular}{lcccc}
$H$ & 10.45 & $(0.479)$ & 10.1 & $(0.339)$ \\
$p_{\text {recall }}$ & 0.25 & $(0.109)$ & 0.70 & $(0.078)$ \\
$p_{\text {store }}$ & 0.75 & $(0.175)$ & 0.25 & $(0.039)$ \\
$\alpha$ & 0.735 & \multicolumn{2}{c}{0.735} & \\
$p$ & \multicolumn{2}{c}{$(t)$}
\end{tabular}

Treatment 2

$\begin{array}{lcccc}H & 15.15 & (0.520) & 11.50 & (0.919) \\ p_{\text {recall }} & 0.50 & (0.121) & 0.70 & (0.078) \\ p_{\text {store }} & 0.60 & (0.133) & 0.25 & (0.039) \\ \alpha & 0.705 & & 0.705 & \\ p & & \left(t, \bar{a}\left(\theta^{\prime}\right)\right) & \end{array}$

Treatment 3

\begin{tabular}{lcccc}
$H$ & 12.45 & $(0.742)$ & 12.75 & $(0.872)$ \\
$p_{\text {recall }}$ & 0.70 & $(0.139)$ & 0.70 & $(0.078)$ \\
$p_{\text {store }}$ & 0.20 & $(0.052)$ & 0.25 & $(0.039)$ \\
$\alpha$ & 0.87 & & 0.87 & \\
$p$ & \multicolumn{3}{c}{$\left(t, \bar{a}\left(\theta^{\prime}\right), i d\left(\theta^{\prime}\right)\right)$}
\end{tabular}

Other Estimated Parameters

None

None

See Camera

See

\& Casari (2009) Appendix A

\# Parameters

Mean Sq. Error

15

0.00091

0.00129

68

25

Indiv. Fixed Effects?

No

No

0.0022

0.0289

Cycle Fixed Effects?

No

No

Yes

No

Predicted Values

Fig. 4(b)

Fig. 4(c)

Fig. 4(d)

Fig. 4(e)

\section{Notes:}

Bootstrapped standard errors are given in parantheses.

All estimates provided here are significant at the 1 percent level.

$\alpha$ and $p$ are set by theory and are not estimated. Only $H, p_{\text {recall }}$, and $p_{\text {store }}$ are estimated.

In the definition of $p$,

$$
\begin{aligned}
& t \text { is the time period, } \\
& \bar{a}\left(\theta^{\prime}\right) \text { is the average cooperation rate of opponent } \theta^{\prime}, \\
& i d\left(\theta^{\prime}\right) \text { is a string of dummy variables that indicate the identity of opponent } \theta^{\prime} .
\end{aligned}
$$

$p_{\text {recall }}$ and $p_{\text {store }}$ are constrained to be identical across treatments in the Constrained CBSA. 
Fig. 4: Cooperation Rates over Time: Experimental Data Versus Alternative Models.

(a) Human Data: Camera and Casari (2009)

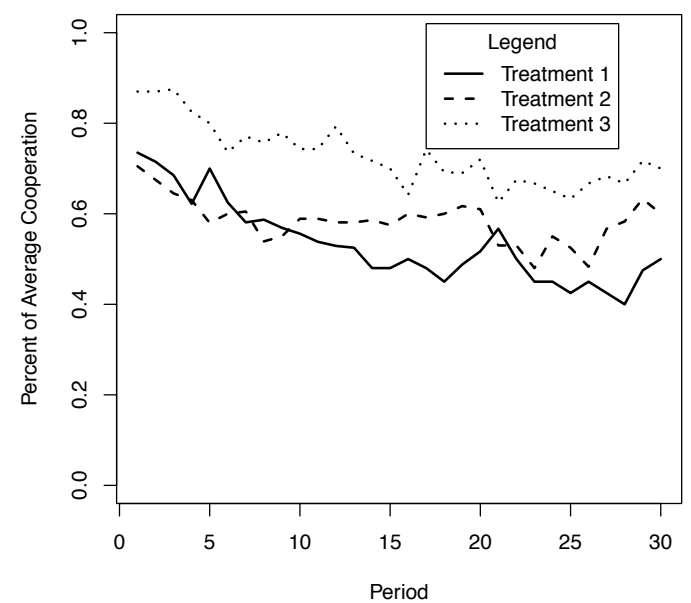

(b) Simulated Data: CBSA. Benchmark model.

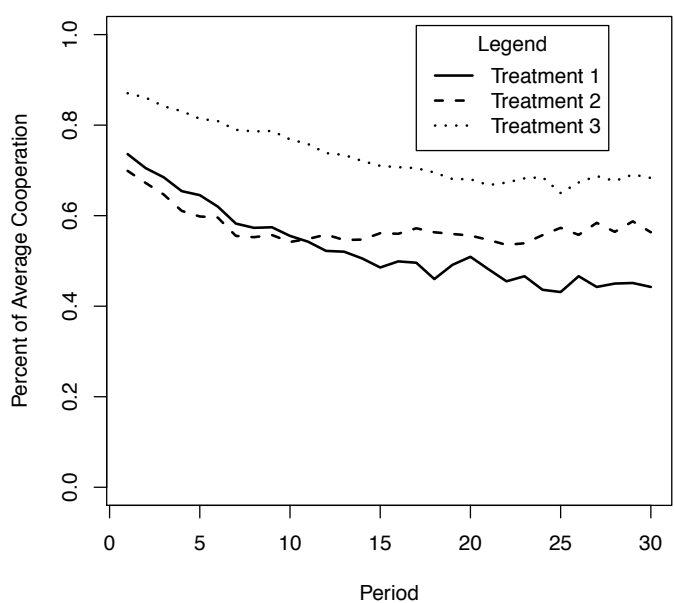

(d) Simulated Data: Full Probit, Camera and Casari.

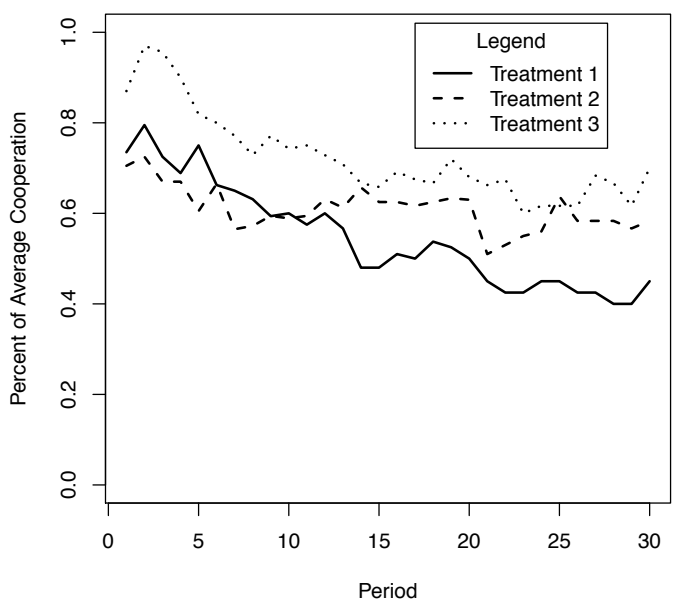

(c) Simulated Data: CBSA. Constrained Alternative.

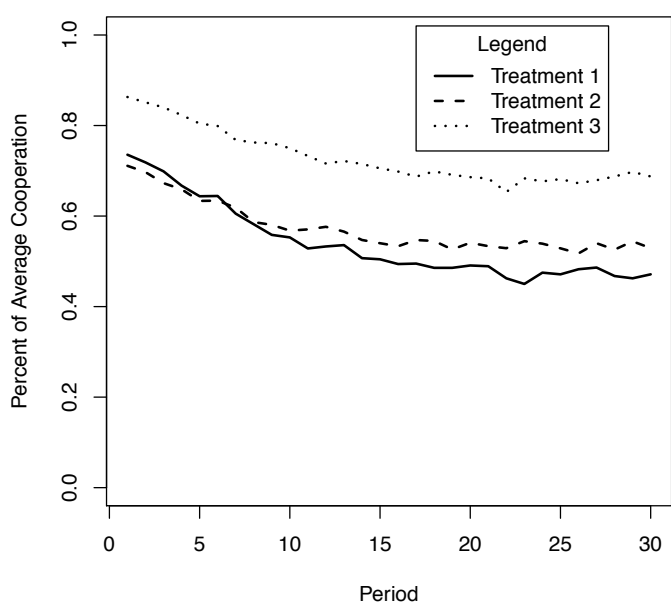

(e) Simulated Data: Constrained Probit.

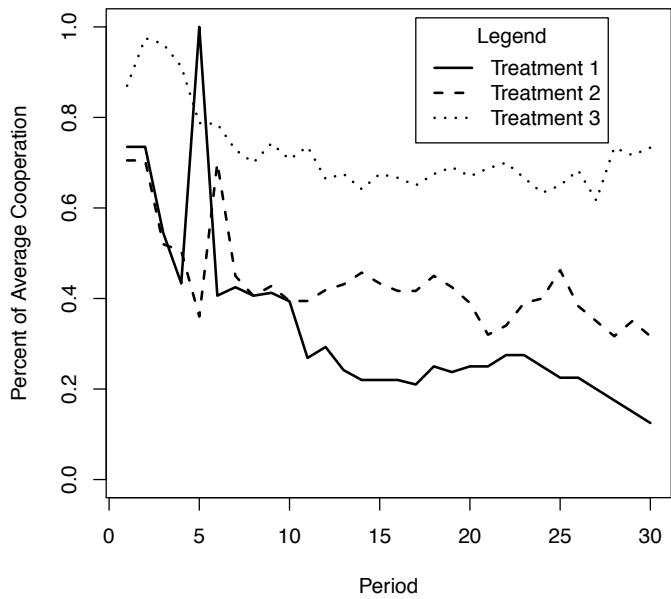


Fig. 5: Cooperation Rates over Time: Comparison by Treatment.

(a) Treatment 1: Private Monitoring

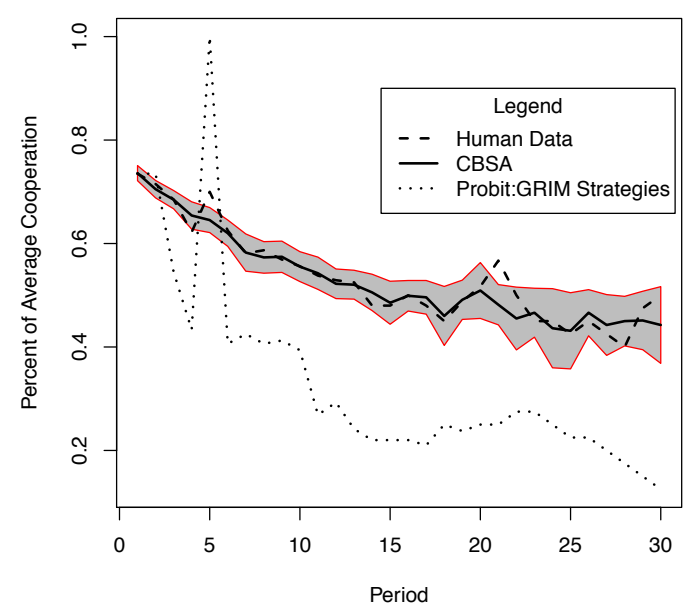

(b) Treatment 2: Anonymous Public Monitoring

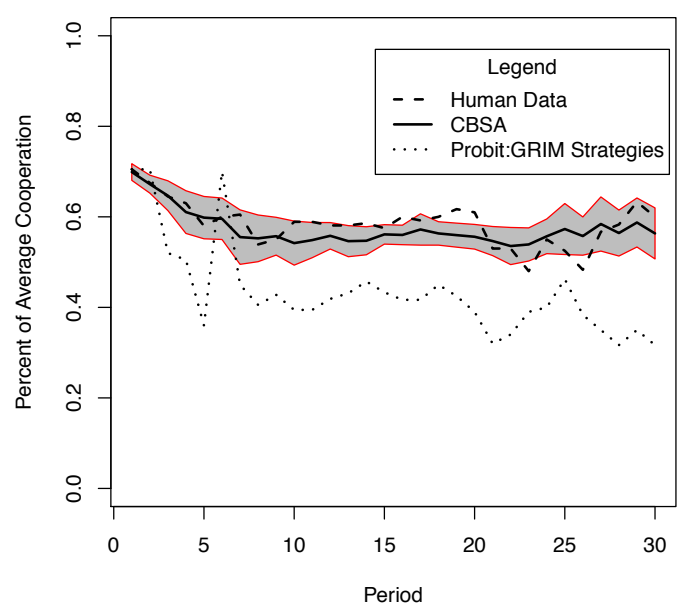

(c) Treatment 3: Non-Anonymous Public Monitoring

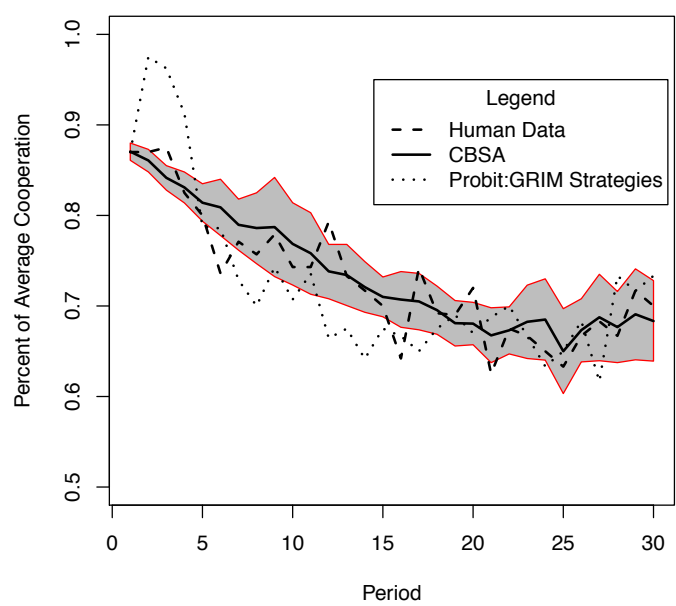

Note: The gray shaded areas depict the $95 \%$ confidence intervals of the prediction of the baseline CBSA model.

compares favorably to the Probit. Fourth, we interpret the estimated parameters from the Benchmark and Constrained CBSA models and establish results (3) and (4) above. ${ }^{25}$

\subsection{Model Descriptions}

The Benchmark CBSA has 15 parameters, which corresponds to five per treatment. For each treatment, two parameters are chosen according to theory and three are estimated. The parameters chosen according to theory are the definition of the problem vector $p$ and the likelihood of choosing cooperate when indifferent, $\alpha$. The estimated parameters are: $H$, the aspiration level; $p_{\text {recall }}$, the probability that a given case is recalled from memory; and $p_{\text {store }}$, the probability that a given case is written to, or stored in, memory.

\footnotetext{
${ }^{25}$ Camera and Casari provide their own interpretation of the Probit parameter estimates, so there is no need to reproduce it here.
} 
The Constrained CBSA is an alternative specification of CBSA. It comes from the following observation: given the fact that data were provided to the subjects of the experiment in all treatments in an identical way, perhaps memory is written to and accessed in an identical away across treatments. The Constrained CBSA formalizes this hypothesis by constraining that the probability of recall $p_{\text {recall }}$ and the probability of storage $p_{\text {store }}$ to be the same across all three treatments. (The aspiration level $H$, the initial rate of cooperation $\alpha$, and the definitions of the problem vector vary across treatments.) As a consequence, the Constrained CBSA has 11 parameters total, only 5 of which are estimated.

The Full Probit refers to the Probit analysis which appears in Camera and Casari (2009), Table 4, page 994. Camera and Casari propose three strategies players might use, and their Probit attempts to empirically identify the relative importance of these strategies in determining behavior. The three strategies are: reactive strategies, global strategies, and targeted strategies. Reactive strategies are: choosing to defect after one's opponent defects. Global strategies are: choosing to defect when any player in the economy defects. Targeted strategies are: choosing to defect against players who have defected against oneself, but ignoring defections against others. Each of these strategies is associated with a lag of one to five periods after a subject experiences a defection, so that the marginal response from a defection can change over those five periods. Camera and Casari's Probit regression is designed to identify the marginal effects of these different strategies, where the binary outcome variable is the choice to cooperate and observations are people-periods. The probit also includes individual and cycle fixed effects, for a total of 68 estimated parameters.

We created the Constrained Probit as a variant to the Camera and Casari Probit. It is the same as the Full Probit, except that there are no cycle and individual fixed effects. The reason for the Constrained Probit is to make a fair comparison to CBSA along the following dimension: because CBSA does not fit parameters for different cycles and individual subjects, it can predict out-of-sample in its current form. However, the Full Probit allows independent fitting for individuals. These individual fixed effects would not be available for predicting out-of-sample. So the Constrained Probit is, in some sense, the best-fitting Probit which allows for the same freedom to predict out of sample as does CBSA. (The same reasoning could be applied to out-of-sample prediction for any econometric model. It guards against over-fitting.) The Constrained Probit has 25 estimated parameters. The results of the Constrained Probit can be found in the Appendix A.

For all four models-the two CBSAs and the two Probits-we construct the aggregate level of cooperation in each time period $t$ :

$$
C L_{t}=\sum_{i \in I_{t}} \frac{a_{i, t}}{N_{t}}
$$

Where $I_{t}$ is the set of subjects still playing at time $t, N_{t}$ is the number of subjects still playing at time $t$, and where $a_{i, t}$ equals 1 if a player $i$ chooses Cooperate and 0 if she chooses Defect. In the experiment, $N_{t}$ varies with treatment and time period. In the CBSA results, $N_{t}$ is as high as 4,000 (we simulate 1,000 economies, each with four players, and as supergames end in the data, this value falls). To generate $C L_{t}$ in the Full and Constrained Probit, we use the predicted value of the dependent variable, $\hat{Y}_{i}$, for each agent in the data. $\hat{Y}_{i}$ is the probability of cooperating. We record a binary value of 1 if $\hat{Y}_{i} \geq 0.50$ and 0 if $\hat{Y}_{i}<0.50$. Then we construct the average cooperation level of all observations per period across economies as described above. 
Table 1 summarizes both the quantitative fit, MSE, and one measure of model complexity, the number of estimated parameters. It also depicts the bootstrapped standard errors.

With regard to quantitative fit, the order of Mean Squared Error (MSE) is:

$$
\text { Benchmark CBSA }<\text { Constrained CBSA } \leq \text { Full Probit }<<\text { Constrained Probit }
$$

The Benchmark CBSA is a better fit than any other model available by a significant degree. The next best is fit the Constrained CBSA, although the Full Probit is close (only 22\% larger MSE). The Constrained Probit performs far worse than the others: its MSE is over 30 times that of the Benchmark CBSA and ten times that of the Full Probit.

These results are particularly striking when one considers the model complexity. The Full Probit is worse at explaining the average cooperation rates despite having four times the number of free parameters. The Full Probit, as opposed to the Constrained Probit, also takes advantage of individual fixed effects, which CBSA does not allow (CBSA assumes that all agents are ex-ante identical and differ only because of experiences over the course of the run.) Dropping individual fixed effects in the Constrained Probit reduces the model complexity to only about twice that of the Benchmark CBSA, but at the cost of large amounts of predictive power.

\subsection{Model Comparison: Qualitative Fit (Results 1 and 2)}

Figure 4 visually depicts the actual average cooperation rates in the experiment versus the four models' predicted average cooperation rates. Figure 4(a) depicts the average cooperation rates over time in the human trials as found by Camera and Casari. Figures 4(b) and 4(c) depict the average predicted cooperation rates over time in the CBSA models; first the Benchmark, then the Constrained. Figures 4(d) and 4(e) depict the predicted average cooperation rates over time which arise from the Probit models; first the Full, then the Constrained.

Consider the following observations about the human cooperation rates as seen in Figure 4(a). First, Treatment 3 does not overlap with Treatments 1 and 2 and instead lies strictly above it for the entirety of the thirty periods. Second, Treatment 1 involves somewhat more cooperation than Treatment 2 in early periods, until some time between rounds 5 and 10 , where Treatment 2 begins to involve more cooperation. Treatment 2 continues to involve more cooperation until the end, with the exception of a brief time around Period 20. Third, average cooperation rates appear to settle into their long-run averages around period fifteen or so. There is a possible upward drift in the three treatments, suggesting if the experiments were to have gone on longer, perhaps average cooperation rates would be $\mathrm{u}$-shaped. ${ }^{26}$

The CBSA predicted cooperation rates, Figures 4(b) and 4(c), match the human data qualitatively quite well. First, Treatment 3 is strictly above the other treatments for the entirety of the run. Second, Treatment 1 has an early lead in cooperation over Treatment 2, but switches places around between periods 5 and 10, as in the human data. (It also ignores the brief reversal around Period 20, which looks like noise.) The crossover occurs a bit too early in the Constrained CBSA. Third, average cooperation rates settle into their long run averages fairly early, although apparently earlier than the human data (more likely by Period 10 to 15). There is also a possible upward drift in all treatments near the end, par-

26 This is also predicted in the long run by CBSA; see Section 5.4 below. 
ticularly in Treatment 2. The upward drift in Treatment 2 is perhaps too pronounced in the Constrained CBSA.

The Full Probit, Figure 4(d), matches the human data somewhat well: Treatment 3 is largely above the other two treatments, Treatment 1 and 2 switch as they do in the human data, with overlap seen in the human data. Also, there appears to be some possible upward drift in all Treatments near the end. The most significant way that the Full Probit matches the human data better than the CBSA data is, Treatment 1's spike around period 5. The Full Probit has this spike (and the Constrained Probit has it in an even more exaggerated way). CBSA has this spike only mildly (and, in fact, CBSA seems significantly less noisy than the Probit). In the authors' opinion, this brief spike is likely just noise in the human data and not a meaningful feature to attempt to match. So the extent to which the Full Probit 'overfits' on such features, it would suggest that CBSA is a better qualitative fit. On the other hand, if one believes that the period 5 spike in the human data is not noise, that the Full Probit picked it up is to its favor.

This spike does cause the Constrained Probit to violate the ordering that Treatment 3 has higher cooperation rates than Treatments 1 and 2 over the whole trial. Although the Full Probit matches the trajectory and final average cooperation rates fairly well, under the Constrained Probit, the average cooperation rates for the Probit under Treatments 1 and 2 falls much faster than either the human data or the benchmark CBSA, resulting in a final average level of cooperation much lower than the observed level of cooperation. Finally, in Treatments 1 and 2, there is definitely no upward drift seen, although possibly in Treatment 3 .

Also consider Figure 5, in which, using the bootstrap method we calculate the standard errors of the predicted cooperation rate for the CBSA benchmark model and display the $95 \%$ percent level confidence intervals, shown as the gray area around the CBSA prediction. This provides us with the measure of variation in prediction which appears to fit the experimental data quite well in all three treatments. This provides further qualitative evidence that CBSA can consistently predict the average cooperation rates in the empirical data over time.

\subsection{CBSA: Interpretation of Estimated Parameters (Results 3 and 4)}

We can establish, using bootstrapped standard errors, that all of the point estimates in the CBSA models are highly significant at the 1 percent level. Furthermore, the individual estimates from the treatments are different from each other in most cases. There are significant differences between the estimates in the benchmark CBSA of $H$ between treatments at the 5 percent level, found by using Z-test. The estimates of $p_{\text {store }}$ are not statistically different from each other at the 10 percent level across almost all treatments. The $p_{\text {recall }}$ is statistically different when comparing treatment 3 to the other treatments. The estimates of $p_{\text {recall }}$ and $p_{\text {store }}$ do not have a consistent ordering.

Here we interpret the values of the estimated parameters, $p_{\text {recall }}, p_{\text {store, }}$ and $H$, in the best-fitting CBSA models and establish the results three and four: (3) that the aspiration levels are 'reasonable' and (4) the memory parameters circumstances with more details are easier to recall. ${ }^{27}$ In the course of the presentation we investigate these claims with the aid of some robustness tests.

Result 3: The aspiration level $H$ represents a target payoff level that the agent seeks in the PD Stage game (Table 1). For the Benchmark and Constrained CBSAs, the fitted values for all three treatments are above the $(D, D)$ payoff $(10)$ but below the $(C, C)$ payoff $(25)$. This suggests that agents hope to

\footnotetext{
27 We do not interpret $p$, the problem set, and $\alpha$, the probability of cooperating when indifferent, because they are set by theory; therefore, their values are not a "finding" and are therefore it is not appropriate to interpret them as one would estimated parameters. Please see Section 3 for how those parameters were chosen.
} 
do better than the stage game Nash equilibrium but are not confident that they will. Aspiration levels can also be interpreted as the satisficing payment required for a subject to stop searching for a better outcome. This leads to an interpretation of the aspiration level as a weighted average of the two symmetric outcomes, $(C, C)$ and $(D, D) \cdot{ }^{28}$ In the benchmark model, this interpretation implies that the agent only hopes for the mutual co-operation outcome about $3 \%$ of the time in Treatment $1,34 \%$ of the time in Treatment 2, and $16 \%$ of the time in Treatment 3. (The values are similar in the Constrained CBSA.) The aspiration level is higher in 2 than in 1, and higher in 3 than in 1. This is consistent with the interpretation that agents hope more monitoring will increase cooperation. However, that interpretation is somewhat undercut by the fact that the aspiration level is higher in Treatment 2 than in Treatment 3.

We run a robustness test by varying the aspiration level to examine the implications of behavior in our model. In the Benchmark CBSA, when the aspiration level $H$ is set to be higher than achievable, 31 , the mean squared error increases about twenty eight-fold, to 0.022 . When it is constrained to be below the worst payoff, 4 , the MSE increases about twenty five-fold, to 0.025 . The fact that reasonable aspiration values provide a better fit than 'unreasonable' aspiration values should be interpreted as a vote in favor of CBSA being the correct model specification.

It is worth considering the behavior one expects with different aspiration levels. As mentioned in the description of CBDT, aspiration levels function as a satisficing level. This can be seen in the functional form of case-based utility, which awards a payoff of $[u(r)-H]$ for a case in memory which yields an outcome $r$. Consider a new experienced case $(p, a, r)$. If $u(r)$ falls short of $H$, this new case would tend to discourage action $a$ in circumstance $p$ (or other, similar circumstances), while if $u(r)$ exceeds $H$, this case encourages this same action in such circumstances. ${ }^{29}$

This implies that aspiration levels have the following behavioral implications: a very low aspiration level yields quick 'convergence' in behavior for all circumstances that is heavily influenced by early choices. A very high aspiration level yields continuous 'searching,' so agents are seen vacillating between defection and cooperation for the same or similar circumstances. An intermediate aspiration level, then, leads to some searching. For circumstances that are predictive of opponent's behavior and therefore of the payoffs associated with ones own actions, the agent may learn which action is appropriate (yields the highest available payoff) and circumstances that are not predictive (or predictive of only low-payoff-outcomes) will prompt the agent to continue to search.

Result 4: The memory probabilities $p_{\text {recall }}$ and $p_{\text {store }}$ can be interpreted as follows: A low $p_{\text {recall }}$ implies the relevant limitation is cognitive capacity, while a low $p_{\text {store }}$ implies that the relevant limitation is memory space. These parameters vary across the three treatments in the Benchmark CBSA, and are constrained to be identical in the Constrained CBSA.

Let us consider the ordering of $p_{\text {recall }}$ across treatments in the Benchmark CBSA. In recall probability $p_{\text {recall }}$, this is the ordering: $p_{\text {recall }}\left(\right.$ Treat $\left._{1}\right)<p_{\text {recall }}\left(\right.$ Treat $\left._{2}\right)<p_{\text {recall }}\left(\right.$ Treat $\left._{3}\right)$. This suggests as the length of the problem vector (i.e. available information) increases, the probability of recall increases. This seems counter-intuitive: the longer the problem vector, the more information must be recalled in the future. Presumably more information is more difficult to store and recall than less information, which would make it harder, not easier, to recall. On the other hand, adding information that is relevant to the problem could have the opposite effect. For example, if one has access to the opponent's ID, it may take less effort to store and recall cases from earlier periods because there is a 'marker' to attach those

\footnotetext{
28 One could also interpret the value as being induced by some 'hope distribution' over all four payoff values, $5,10,25$, and 30 .

29 The agent-based modeling literature describes these two behaviors as 'exploration' versus 'exploitation,' so aspiration levels determine the switch from exploration to exploitation.
} 
memories to: this ID variable. In any case, the apparent empirical fact is that the recall probability is inversely related to the length of the problem vector.

Although in previous work (Pape and Kurtz, 2013), it was found that $p_{\text {recall }}$ was found to be smaller than $p_{\text {store }}$, that does not to hold in this experiment, where we find that Treatment 1 and 2 have $p_{\text {recall }}$ less than $p_{\text {store }}$, but Treatment 3 and the constrained CBSA have the reverse.

For storage probability $p_{\text {store }}$, the ordering suggests that the probability of storage decreases the length of the problem vector: $p_{\text {store }}\left(\right.$ Treat $\left._{3}\right) \leq p_{\text {store }}\left(\right.$ Treat $\left._{2}\right)<p_{\text {store }}\left(\right.$ Treat $\left._{1}\right)$. However, this set of orderings is not statistically significant at the $10 \%$ level.

Fig. 6: Cooperation Rates: Benchmark in the Long Run versus Perfect Memory

(a) Perfect Memory

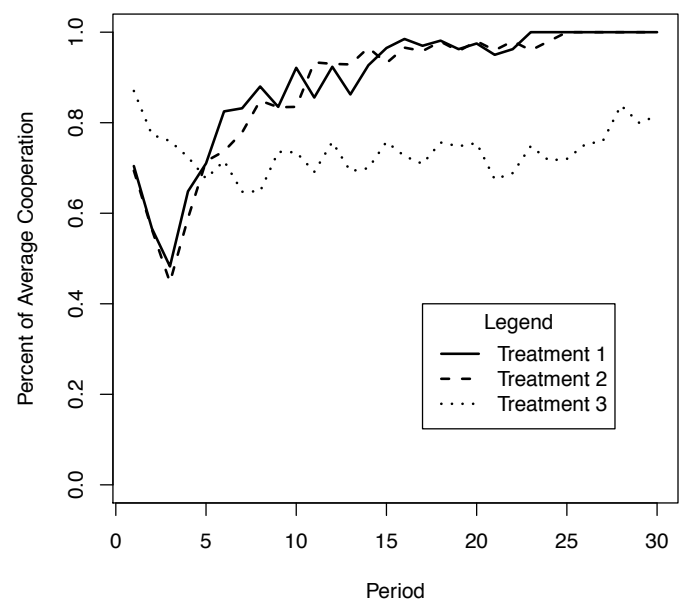

(b) Benchmark Model in Long Run

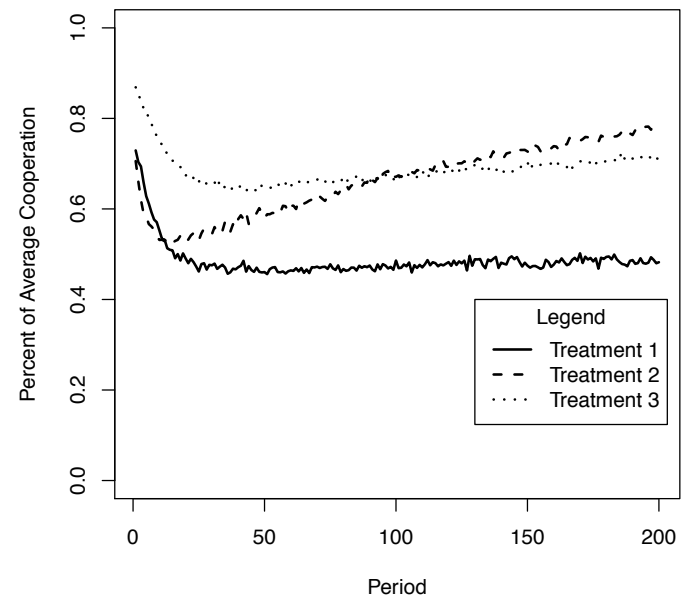

Next we consider the implications of perfect memory in the CBSA. When we only consider perfect memory, i.e. $p_{\text {recall }}=p_{\text {store }}=1$, we find that the MSE worsens significantly. When memory is constrained to be perfect, the MSE increases about one hundred-fold, to 0.10 . This suggests that imperfect memory may be more important than reasonable aspiration values. In Figure 6(a), we see that in Treatments 1 and 2, the downward trend quickly reverses toward perfect cooperation, and even Treatment 3 shows some upward drift near the end of the displayed timeframe. (The other parameter values are kept at the level set in Table 1). We compare these results to long run results of the benchmark model in Figure 6(b). In this figure, the benchmark model is run for 200 periods. We see that even under imperfect memory, cooperation heads toward perfect cooperation in Treatment 2, with some upward tendency in Treatment 3 as well. We also find that for an increase in memory over those values found in Table 1 (not shown here), Treatments 1 and 3 also head toward perfect cooperation in this timeframe. This pattern is robust to lowering the probability of cooperation when indifferent, so this is not a product of over-selection under indifference. Instead, what is happening is that agents eventually 'find' the cooperative outcome and then, as they go into the future, the early events that led to defection fade into the past and cooperation wins over. When there is perfect memory, this effect occurs too early to be consistent with the human data.

This observed effect makes an out-of-sample prediction: we predict that among human players, if game play was allowed to continue we would expect that the leveling off would eventually turn around 
to increasing cooperation in the long run, most strongly with Treatment 2 . This suggests it would be valuable to do repeated Prisoner's Dilemma experiments for longer time spans (i.e. higher continuation probabilities) to see whether this out-of-sample prediction is achieved.

\section{Discussion}

In this section, we discuss the broader implications of these results. To the author's knowledge, this is the first application of case-based decision theory to a strategic/game-theoretic context. What is the appropriateness of such an application? After all, case-based decision theory is developed for individual choice; is it appropriate to view game play as merely another form of decision-making? And, given this match in this strategic setting between case-based decision theory - as implemented through CBSAand human choice, what have we learned about case-based decision theory? What have we learned about learning in games?

Let us consider strategic choice problem as a decision theory problem in the expected utility paradigm. In order to do so, one must define the state space. The state space in this setting is the set of possible strategies (complete contingent plans) that one's opponent (and Nature) might use. From this perspective, the mapping of a game into the expected utility framework is straight-forward. Consider von Neumann and Morgenstern in The Theory of Games and Economic Behavior: they transform an arbitrarily complicated finite game into a game in which each player makes one move which specifies all of their contingent plays; each player submits this move to an umpire who then works out the payoffs for all players. This results in a single expression for the player's probability-weighted payoff, "his "mathematical expectation' of the outcome (von Neumann and Morgenstern, 1944, Section 11.2.3)." They continue: "The player's judgement must be directed solely by this 'mathematical expectation,'- -because the various moves [of other players and Nature] are completely isolated from each other." (The mathematical expectation referred to here is expected utility as von Neumann and Morgenstern define it (See von Neumann and Morgenstern, 1944, Section 5.2.2).) The only remaining issue to determine choice is the probability distribution over other players' strategies. The zero-sum analysis of von Neumann and Morgenstern and Nash's subsequent work (Nash, 1950, 1953) can be thought of as a search of this space for 'reasonable' priors. This historical note justifies the approach of viewing strategic choice as another form of individual choice. Furthermore, and in justification of case-based decision theory specifically, the fact that the state-space representation is well-defined mathematically does not imply that it is intuitive for actual human players. If it is not intuitive for actual human players, then the objection of the state-space representation described by Gilboa and Schmeidler in their original work (Gilboa and Schmeidler, 1995): if the state-space is not intuitive for agents, then it might not be how agents actually represent their problem, so the conclusions of that theory may not be helpful in understanding how humans actually choose. Saying the reverse, finding a representation that is more intuitive for people may lead to a theory that is better at predicting how people actually choose.

Along those lines, consider the theoretical (vs. empirical/experimental) game theory literature of learning in games. The basic problem faced by researchers in this literature is that for an agent to "learn" the strategy of her opponent, one of two things must hold: she must have some a priori rule to extrapolate from past actions to future actions or some a priori restriction on the set of possible strategies that are allowable. Why? Nachbar (1997) makes a concise formal argument: "Formally, if a player can learn to predict the continuation path of play then, in particular, the player can learn to predict the distribution over play in the next period. Let a one-period-ahead prediction rule be a function that, for each history, 
chooses a probability distribution over the opponent's stage game actions. [...] [F]or any one-period-ahead prediction rule, whether or not derived via Bayesian updating, there exists an opposing strategy that does "the opposite." [...] [S]o there is no one-period-ahead rule that is asymptotically accurate against all strategies (Nachbar, 1997, p. 227, footnote 3.)." From this perspective, the learning-in-games literature can be thought of as proposing different extrapolation rules and/or restricted subsets. Nachbar (1997), for example, proposes a set of 'conventional' strategies, and supposes that (it is common knowledge that) agents have a lexicographic preference for conventional vs. unconventional strategies. Another prominent example is Fudenberg and Kreps $(1993,1995)$, who suppose that opponents' play corresponds to historical frequencies of past play ("fictitious play") which leads to so-called self-confirming equilibria. From this perspective, case-based decision theory is simply one such extrapolation rule. Why choose a case-based rule? The first justification is, of course, the empirical success of Pape and Kurtz (2013) explaining human learning in another setting. Another justification, however, relates directly to this basic problem that Nachbar describes so effectively. In the early history of the philosophy of learning, Hume (1748) introduced the 'induction problem.' Gilboa and Schmeidler take as some inspiration of case-based reasoning Hume's observation that "[f]rom causes which appear similar we expect similar effects. This is the sum of all of our experimental conclusions (Hume, 1748)." The reference to similarity is the inspiration of Gilboa and Schmeidler to formally incorporate 'similarity' in their model. Nachbar's observation that there must be some a priori extrapolation rule or a priori restriction on the set possible ideas in order to learn from experience is essentially a restatement of the induction problem as introduced by Hume. Now, Hume's point is that, inferring from past events to future events falls short of 'true knowledge' as one might want from a philosophical point of view; that is not the point here, however. The point here is to take Hume's claim as a hypothesis regarding human learning behavior: that actual people extrapolate from past events to future events using similarity. This is a strong, historically-rooted justification for using a case-based approach to model human behavior in learning-in-games. Moreover, since we find that case-based decision theory provides a good match for human learning behavior, it suggests that the development of a formal equilibrium concept along these lines - a case-based equilibrium - could be a fruitful endeavor. Perhaps the most profitable direction would be to investigate the relationship between learning implied by case-based decision theory and fictitious play proposed by Fudenberg and Kreps. ${ }^{30}$

One vein of experimental/empirical game theory literature regarding learning in games involves explaining human game-play using reinforcement learning (Roth and Erev, 1995b; Bereby-Meyer and Erev, 1998; Erev and Roth, 1998; Erev, Bereby-Meyer, and Roth, 1999; Erev and Roth, 2001). The Roth, Erev, and Bereby-Meyer papers measure success in explaining human data by considering the mean squared deviation between average results of simulated and observed data, as we do here. This supports the methodology we describe in Section 4. In reinforcement learning, each agent keeps track of a weight (value) for each action which indicates her proclivity to take that action. When she takes an action and it delivers a payoff which exceeds some reference level, she increases the weight on that action, and when it falls short, she decreases the weight on that action. Reinforcement learning can be made more complex: for example, the reference level can vary over time and be endogenous to experience in some manner, or the agent can have multiple sets of action weights, so that different circumstances invoke different action weights. Reinforcement learning is related but distinct from case-based learning. For example, in both theories, reference levels or aspiration levels play a key "sign-changing" role. The major difference is: first of all, CBDT does not collapse history into a single set of weights, so CBDT allows for history to be re-examined later in the agent's experience. Also, CBDT has similarity, which seems to be a critical

30 We intend to develop this relationship in future work. 
component to getting human learning behavior correct. ${ }^{31}$ Although we do not explicitly compare reinforcement learning vs. case-based decision theory on the Camera and Casari data, following the work of Pape and Kurtz (2013), it can be shown that while 'simple' case-based learning reproduces the ordering of difficulty of human concepts found in the psychological concept learning experiments of Nosofsky and Palmeri (1996), reinforcement learning does not. ${ }^{32}$ Since differences between these approaches can be found, it suggests that we may learn more about case-based learning by a closer comparison between CBSA and reinforcement learning, which we intend to pursue in a future extension.

\section{Conclusion}

In this paper, we use Case-based Decision Theory to explain the average cooperation level over time of the repeated Prisoner's Dilemma among random pairs of people in a small group; the work of Camera and Casari (2009). We are the first to use a method called the Case-based Software Agent and show that CBSA (and therefore CBDT) empirically explains human behavior in a strategic, dynamic, multi-agent setting. This provides a different frame of reference to view the results in the iterated prisoner's dilemma game that incorporates past experiences into explaining sustained cooperative outcomes.

We establish four main results.

First, we find that CBSA predicts human behavior in this strategic and dynamic setting quite well. It has a good quantitative fit, and its predicted outcome variable has the main patterns of the human data without over-fitting. This is a vote in favor of CBSA and CBDT as a explanation of human behavior in this setting.

Second, we find that CBSA compares quite favorably with the Probit from Camera and Casari: it has an arguably stronger qualitative fit, a stronger quantitative fit, and fewer free parameters to achieve this fit. Combined with Result 1, a fairly strong empirical argument can be made that CBSA should be considered seriously as an empirical explanation of human behavior in the repeated Prisoner's Dilemma.

Third, we find best-fitting CBSA aspiration value $H$ implies humans aspire to payoff value above the mutual defection outcome but below the mutual cooperation outcome, which suggests they hope, but are not confident, that cooperation can be achieved. In principle, the best-fitting aspiration values could have fallen into the 'unreasonable' range-greater than the best or lower than the worst possible outcome-which would have implied that CBSA is misspecified.

Fourth, we find evidence that suggests that circumstances with more details are easier to recall. The evidence is that our best-fitting level of probability of recall increase as the experimental treatment vary as to share more information with the agents. The best-fitting parameter values suggest that in treatments where subjects have more public information, such as the ID of the partner, the probability of recall increases.

This paper was predicted in the concluding paragraph of Pape and Kurtz (2013): "This computational implementation of Case-based Decision Theory"-that is, CBSA-"can be calibrated to and tested against human data in any existing experiment which can be represented in a game-theoretic form[.] This suggests a model for future studies. As these studies accumulate, we will learn whether and when Case-based

\footnotetext{
31 Pape and Kurtz (2013) investigate alternative similarity functions, including ones that involve 'no' similarity, and these do not capture the facts regarding relative difficulty of problems that human subjects find.

32 With 1100 simulation runs for each of the six problem category types, case-based decision theory finds the ordering that are observed in humans, with statistically significant differences. (This echoes the results of Pape and Kurtz (2013).) However, with the same number of runs, reinforcement learning finds all problems of equal difficulty (at least, not statistically significantly different in difficulty) and the point estimates do not follow the human ordering. Please see Appendix $\mathrm{C}$ for details.
} 
Decision Theory provides an adequate explanation of human behavior in other decision settings and may also learn which parameters appear to vary by setting and which, if any, remain constant across settings." With more studies like this one, their stated goal may be achieved: "This could lead to a version of CBDT which can be used to simulate human behavior in a variety of economic models." 


\section{References}

Andreoni J, Miller JH (1993) Rational cooperation in the finitely repeated prisoner's dilemma: Experimental evidence. The Economic Journal 103(418):570-585

Axelrod R (1980) Effective choice in the prisoner's dilemma. Journal of Conflict Resolution 24(1):3-25

Ballinger TP, Hudson E, Karkoviata L, Wilcox NT (2011) Saving behavior and cognitive abilities. Experimental Economics 14(3):349-374

Bereby-Meyer Y, Erev I (1998) On learning to become a successful loser: a comparison of alternative abstractions of learning processes in the loss domain. Journal of mathematical psychology 42(2):266286

Bereby-Meyer Y, Roth AE (2006) The speed of learning in noisy games: partial reinforcement and the sustainability of cooperation. The American Economic Review 96(4):1029-1042

Billot A, Gilboa I, Schmeidler D (2008) Axiomatization of an exponential similarity function. Mathematical Social Sciences 55(2):107-115

Bishop YM, Fienberg SE, Holland PW (1975) Discrete multivariate analysis: Theory and Practice. MIT Press, Cambridge, MA

Bleichrodt H, Filko M, Kothiyal A, Wakker PP (2012) Making case-based decision theory directly observable

Bó PD (2005) Cooperation under the shadow of the future: experimental evidence from infinitely repeated games. The American Economic Review 95(5):1591-1604

Bo PD, Fréchette GR (2011) The evolution of cooperation in infinitely repeated games: Experimental evidence. The American Economic Review 101(1):411-429

Brosig J (2002) Identifying cooperative behavior: some experimental results in a prisoner's dilemma game. Journal of Economic Behavior \& Organization 47(3):275-290

Camera G, Casari M (2009) Cooperation among strangers under the shadow of the future. The American Economic Review pp 979-1005

Camerer C, Hua Ho T (1999) Experience-weighted attraction learning in normal form games. Econometrica $67(4): 827-874$

Cappelletti D, Guth W, Ploner M (2011) Being of two minds: Ultimatum offers under cognitive constraints. Journal of Economic Psychology 32(6):940 - 950

Chong JK, Camerer CF, Ho TH (2006) A learning-based model of repeated games with incomplete information. Games and Economic Behavior 55(2):340-371

Cooper R, DeJong DV, Forsythe R, Ross TW (1996) Cooperation without reputation: experimental evidence from prisoner's dilemma games. Games and Economic Behavior 12(2):187-218

Ellison G (1994) Cooperation in the prisoner's dilemma with anonymous random matching. The Review of Economic Studies 61(3):567-588

Ellsberg D (1961) Risk, ambiguity, and the savage axioms. Quarterly Journal of Economics 75(4):643-669

Erev I, Roth AE (1998) Predicting how people play games: Reinforcement learning in experimental games with unique, mixed strategy equilibria. American economic review pp 848-881

Erev I, Roth AE (2001) Simple reinforcement learning models and reciprocation in the prisoners dilemma game. Bounded rationality: The adaptive toolbox pp 215-231

Erev I, Bereby-Meyer Y, Roth AE (1999) The effect of adding a constant to all payoffs: experimental investigation, and implications for reinforcement learning models. Journal of Economic Behavior \& Organization 39(1):111-128 
Friedman JW (1971) A non-cooperative equilibrium for supergames. The Review of Economic Studies pp $1-12$

Fudenberg D, Kreps DM (1993) Learning mixed equilibria. Games and Economic Behavior 5(3):320-367

Fudenberg D, Kreps DM (1995) Learning in extensive-form games I. Self-confirming equilibria. Games and Economic Behavior 8(1):20-55

Fudenberg D, Maskin E (1986) The folk theorem in repeated games with discounting or with incomplete information. Econometrica: Journal of the Econometric Society pp 533-554

Gayer G, Gilboa I, Lieberman O (2007) Rule-based and case-based reasoning in housing prices. The BE Journal of Theoretical Economics (Advances) 7(1)

Gilboa I, Schmeidler D (1995) Case-Based Decision Theory. The Quarterly Journal of Economics 110(3):605-39

Gilboa I, Schmeidler D (1996) Case-based Optimization. Games and Economic Behavior 15:1-26

Golosnoy V, Okhrin Y (2008) General uncertainty in portfolio selection: A case-based decision approach. Journal of Economic Behavior \& Organization 67(3):718-734

Hanoch Y (2002) "Neither an angel nor an ant": Emotion as an aid to bounded rationality. Journal of Economic Psychology 23(1):1-25

Hauk E, Nagel R (2001) Choice of partners in multiple two-person prisoner's dilemma games an experimental study. Journal of conflict resolution 45(6):770-793

Hume D (1748) Philosophical essays concerning human understanding. A. Millar

Kreps DM, Milgrom P, Roberts J, Wilson R (1982) Rational cooperation in the finitely repeated prisoners' dilemma. Journal of Economic theory 27(2):245-252

Kurtz KJ (2007) The divergent autoencoder (DIVA) model of category learning. Psychonomic Bulletin \& Review 14(4):560-576

Kydland FE, Prescott EC (1996) The computational experiment: An econometric tool. The Journal of Economic Perspectives 10(1):pp. 69-85

Love B, Medin D, Gureckis T (2004) SUSTAIN: A Network Model of Category Learning. Psychological Review 111(2):309

Luce RD, Raiffa H (1957) Games and Decisions. WIley, New York

Matsui A (2000) Expected utility and case-based reasoning. Mathematical Social Sciences 39(1):1-12

Miller JH (1996) The coevolution of automata in the repeated prisoner's dilemma. Journal of Economic Behavior \& Organization 29(1):87-112

Monterosso J (2002) The Fragility of Cooperation: A False Feedback Study of a Sequential Iterated Prisoner's Dilemma. Journal of Economic Psychology 23(4):437 - 448

Nachbar JH (1997) Prediction, optimization, and learning in repeated games. Econometrica: Journal of the Econometric Society pp 275-309

Nash J (1950) The bargaining problem. Econometrica: Journal of the Econometric Society pp 155-162

Nash J (1953) Two-person cooperative games. Econometrica: Journal of the Econometric Society pp $128-140$

von Neumann J, Morgenstern O (1944) Theory of Games and Economic Behavior. Princeton University Press, Princeton, NJ

Nosofsky R (1986) Attention, Similarity, and the Identification-Categorization Relationship. Journal of Experimental Psychology: General 115(1):39-57

Nosofsky R, Palmeri T (1996) Learning to Classify Integral-Dimension Stimuli. Psychonomic Bulletin and Review 3:222-226 
Nosofsky R, Gluck M, Palmeri T, McKinley S, Glauthier P (1994) Comparing Models of Rule-Based Classification Learning: a Replication and Extension of Shepard, Hovland, and Jenkins (1961). Memory and Cognition 22:352-352

Ossadnik W, Wilmsmann D, Niemann B (2012) Experimental evidence on case-based decision theory. Theory and Decision pp 1-22, DOI 10.1007/s11238-012-9333-4, URL http://dx.doi.org/10.1007/s11238-012-9333-4

Pape AD, Kurtz KJ (2013) Evaluating case-based decision theory: Predicting empirical patterns of human classification learning. Games and Economic Behavior 82:52-65

Roth AE, Erev I (1995a) Learning in extensive-form games: Experimental data and simple dynamic models in the intermediate term. Games and Economic Behavior 8(1):164-212

Roth AE, Erev I (1995b) Learning in extensive-form games: Experimental data and simple dynamic models in the intermediate term. Games and economic behavior 8(1):164-212

Savage LJ (1954) The Foundations of Statistics. Wiley

Selten R, Stoecker R (1986) End behavior in sequences of finite prisoner's dilemma supergames a learning theory approach. Journal of Economic Behavior \& Organization 7(1):47-70

Shepard R (1987) Toward a Universal Law of Generalization for Psychological Science. Science 237(4820):1317

Shepard R, Hovland C, Jenkins H (1961) Learning and Memorization of Classifications. Psychological Monographs 75:1-41

Simon HA (1957) Models of man; social and rational.

Simon HA, Egidi M, Marris RL (2008) Economics, bounded rationality and the cognitive revolution. Edward Elgar Publishing

Tesfatsion L (2006) Handbook of Computational Economics, vol 2, Elsevier B.V., chap 16

Tyson CJ (2008) Cognitive constraints, contraction consistency, and the satisficing criterion. Journal of Economic Theory 138(1):51-70

Vigo R (2013) The GIST of concepts. Cognition 129(1):138-162

Wooldridge J (2012) Introductory econometrics: A modern approach. Cengage Learning 


\section{A Appendix: Details about the Constrained Probit}

Table 2: Probit Regression on Individual Choice to Cooperate: Marginal Effects

\section{Dependent variable: $1=$ cooperation, $0=$ defection}

\section{Treatment 1 \\ Treatment 2}

Treatment 3

Private monitoring Anonymous public monitoring Public monitoring (non-anonymous)

\section{Reactive strategies}

Grim trigger

$-0.485^{* * *}$

0.007

$\operatorname{lag} 1$

$0.079^{*}$

0.0434

$\operatorname{lag} 2$

$0.088^{* *}$

0.036

$\operatorname{lag} 3$

0.066

0.047

$\operatorname{lag} 4$

$0.038 *$

0.023

$\operatorname{lag} 5$

0.003

$$
\begin{gathered}
-0.309^{* * *} \\
0.093 \\
-0.052^{* * *} \\
0.003 \\
-0.089^{* * *} \\
0.028 \\
-0.071^{* * *} \\
0.019 \\
-0.062^{*} \\
0.034 \\
-0.069^{* * *}
\end{gathered}
$$

0.046

0.035

$-0.084^{* * *}$

0.037

$-0.175^{* * *}$

0.044

$-0.089^{* * *}$

0.018

$-0.075$

0.075

$-0.031$

0.052

\section{Global strategies}

Grim trigger

$-0.248$

$-0.121^{* * *}$

0.171

0.019

$\operatorname{lag} 1$

$\operatorname{lag} 2$

$.194^{* * *}$

0.021

0.065

0.067

0.184

0.026

0.109

0.046

$\operatorname{lag} 3$

$0.191^{* * *}$

0.059

$0.051^{* * *}$

$0.153^{* * *}$

0.019

$\operatorname{lag} 4$

$\operatorname{lag} 5$

$$
0.032
$$

0.003

$0.131^{* * *}$

0.034

$-0.027$

0.034

0.067

\section{Targeted strategies}

Grim trigger

$\operatorname{lag} 1$

$\operatorname{lag} 2$

$\operatorname{lag} 3$

$\operatorname{lag} 4$

$\operatorname{lag} 5$

Sample Size

3,520$$
0.408^{* * *}
$$$$
0.039
$$$$
-0.062^{* * *}
$$$$
0.017
$$$$
-0.079 * * *
$$$$
0.033
$$$$
-0.034
$$$$
0.024
$$$$
-0.062^{* * *}
$$$$
0.007
$$$$
-0.079 * * *
$$$$
0.009
$$

4,280

Similar to the Camera and Casari (2009) paper, this is a Probit regression to identify the marginal effect these different strategies. 2 shows the results of the different strategies from the 'constrained Probit,' which differs from the one in Camera and Casari (2009) only in that individual and cycle fixed effects are excluded. To make clear the construction of the variables included: the grim trigger is coded as 1 for all periods following a defection. The lag variables are to control for the five 
following periods after a defection. The lag 1 contains a 1 for the first period after a defection by an opponent but zero for all other periods, and a lag 2 contains a 1 for the second period after a defection by an opponent. If the player chooses to defect after observing a defection by an opponent then the expectation would be a negative coefficient on at least one of the grim trigger or lags.

\section{B The explicit Problem-Result Map corresponding to Camera and Casari (2009)}

Fig. 7: The Camera and Casari Prisoner's Dilemma Experiment PRM

Input: act $a$, problem $p$, characteristics $\theta$.

1. Find action of opponent $a(\theta)$.

2. Set result $r$ according to the game depicted in Figure 1, with actions $(a, a(\theta))$.

3. Next opponent is chosen $\theta^{\prime}$.

4. Set next problem $p^{\prime}$, by constructing the vector:

$$
p^{\prime}= \begin{cases}(t), & \text { if Treatment } 1 \\ \left(t, \bar{a}\left(\theta^{\prime}\right)\right), & \text { if Treatment } 2 \\ \left(t, \bar{a}\left(\theta^{\prime}\right), i d\left(\theta^{\prime}\right)\right), & \text { if Treatment } 3\end{cases}
$$

Where:

- $t$ is the time period,

- $\bar{a}\left(\theta^{\prime}\right)$ is the average cooperation rate of opponent $\theta^{\prime}$, and

- $i d\left(\theta^{\prime}\right)$ is a string of dummy variables that indicate the identity of opponent $\theta^{\prime}$

Output: result $r$, problem $p^{\prime}$, characteristics $\theta^{\prime}$.

\section{Case-based Decision Theory versus Reinforcement Learning}

Table 3: CBSA versus Reinforcement Learning: Nosofsky and Palmeri '96

\begin{tabular}{cc||cccccc} 
& & I & IV & III & V & II & VI \\
\hline \hline CBSA & Mean & 3.65 & 9.00 & 6.77 & 6.25 & 7.87 & 9.50 \\
& Std. Dev. & 1.12 & 1.88 & 1.35 & 1.93 & 1.48 & 1.22 \\
\hline \multirow{2}{*}{ RL } & Mean & 13.39 & 13.21 & 13.38 & 13.48 & 13.47 & 13.39 \\
& Std. Dev. & 2.70 & 2.80 & 2.78 & 2.74 & 2.60 & 2.62 \\
\hline
\end{tabular}

Table 3 contains the results from CBSA versus Reinforcement Learning on the six canonical learning problems tested in the concept learning literature starting with Shepard et al (1961). CBSA was tested against these types in Pape and Kurtz (2013). Standard CBSA with perfect memory and the standard similarity function was tested against reinforcement learning for these six problems and compared against the data from Nosofsky and Palmeri (1996). The ordering of the columns, $I<I V<I I I<V<I I<V I$, indicates the relative difficulty of the problems as humans find it (in the data by Nosofsky and Palmeri). Each cell contains 1100 simulation runs. Note that the order of the means of the CBSA results match the human ordering and that the order of the means of RL do not. Moreover, the ordering of CBSA is significant (all neighboring pairs pass t-tests at the $1 \%$ level). We believe this is attributable to the similarity function that CBSA has that allows extrapolation from some problems to others. 